\title{
A projection-based approach for the derivation of the floating frame of reference formulation for multibody systems
}

Received: 29 May 2018 / Revised: 27 August 2018 / Published online: 25 October 2018

(C) The Author(s) 2018

\begin{abstract}
The reduction in the number of coordinates for flexible multibody systems is necessary in order to achieve acceptable simulation times of real-life structures and machines. The conventional model order reduction technique for flexible multibody systems is based on the floating frame of reference formulation (FFRF), using a rigid body frame and superimposed small flexible deformations. The FFRF leads to strongly coupled terms in rigid body and flexible coordinates as well as to a non-constant mass matrix. As an alternative to the FFRF, a formulation based on absolute coordinates has been proposed which uses a co-rotational strain. In this way, a constant mass matrix and a co-rotational stiffness matrix are obtained. In order to perform a reduction in the number of coordinates, by means of the component mode synthesis, e.g., the number of modes needs to be increased, such that all modes are represented in every possible rotated configuration. This approach leads to the method of generalized component mode synthesis (GCMS). The present paper shows in detail how the equations of motion of the FFRF evolve from the ones of the GCMS by considering rigid body constraint conditions and subsequently eliminating them via an appropriate null-space projection. This approach allows a straightforward, term-by-term interpretation of the FFRF mass matrix and of the generalized gyroscopic forces, which, to the same extent, cannot be deduced from former publications on the FFRF. From a practical point of view, the resulting expressions allow to calculate all inertia coefficients from the constant finite element mass matrix together with standard input data of the finite element model in the course of a preprocessing step. Then, the repeated updates of the FFRF mass matrix and of the gyroscopic forces in the course of time integration involve only simple vector matrix operations of low dimensions. In contrast to previous implementations of the FFRF, no evaluations of extra inertia integrals are required. Consequently, the present formulation can be implemented entirely independent of the related finite element code.
\end{abstract}

\section{Introduction and state of the art}

Machines, cars, planes, and other technical systems experience a continuous growth in performance. The improvements are usually not based on higher speeds and higher power, but performance is measured with respect to reliability, comfort, accuracy and efficiency. Lightweight design requires to model the mechanical parts of such systems as flexible multibody systems, in order to analyse vibrations and stresses.

A flexible multibody dynamics formulation is used to replace a computationally expensive nonlinear finite element model by a simpler representation, making use of the fact that many bodies undergo small deformations

R. Winkler $(\varangle) \cdot$ J. Gerstmayr

Institute of Mechatronics, University of Innsbruck, Technikerstrasse 13, Innsbruck 6020, Austria

E-mail: Robert.Winkler@uibk.ac.at

Tel.: +43-512-50762752

J. Gerstmayr

E-mail: Johannes.Gerstmayr@uibk.ac.at

Tel.: +43-512-50762750 
and small strains only. In addition, an appropriate method needs to be applied, which can handle joint reactions at the bodies. The conventional approach to model small deformation flexible bodies is the so-called floating frame of reference formulation (FFRF) [19]. Therein, the bodies are assumed to undergo large rigid body translation and rotation as well as superimposed small deformation. The motion of an arbitrary material point of the flexible body is modelled with relative kinematics, summing up translational, rotational and flexible parts of the motion. The equations of motion of a flexible body can be computed from the kinetic energy, the deformation energy and the action of applied loads, constraints, and other force terms.

At this point there are two choices: The first choice, which is the conventional one in the floating frame of reference formulation, is using coordinates for translational motion of the underlying rigid body, rotational parameters for the description of rigid body rotation, and flexible coordinates for the deformation part. In order to derive the equations of motion for the dynamics of a flexible body, any formulation will lead to a coupling of velocities and accelerations with the chosen coordinates. Therefore, the mass matrix will become non-constant and quadratic terms in the velocities result. Due to the assumption of small deformations, the flexible parts of the mass and the stiffness matrices are constant, which allow to apply straightforward model order reduction methods, such as the component mode synthesis (CMS) method, in order to greatly reduce the number of flexible coordinates from millions to dozens. The second choice, denoted as the absolute coordinate formulation (ACF), does not employ a separation into a motion of a reference frame and an elastic deformation, while using the same assumptions on small deformations [10]. The coordinates in the ACF are the same coordinates as in the conventional, possibly nonlinear, finite element method. In order to reduce the computational efforts, the assumption of small deformations in every flexible body is used to co-rotationally linearize the stiffness matrix of every body. The latter approach is closely related to co-rotational finite element formulations $[1,24]$. Note that in the ACF the rigid body rotation refers to the whole body, whereas in corotational formulations, the rotation can vary across the finite element mesh. The second approach leads to much simpler equations of motion, which can be solved more efficiently compared to the first one. However, model order reduction methods cannot be applied in a straightforward way. This is due to the simple fact that the stiffness matrix is co-rotated and thus non-constant. In [8], it has been shown that the ACF and the FFRF are equivalent and thus lead to the same numerical results. It is noted that the ACF shall not to be confused with the absolute nodal coordinate formulation (ANCF). The relation of the FFRF and the ANCF, focussing on beams undergoing large deformations, has already been studied in $[19,20]$.

Fortunately, there is a straightforward way for a model order reduction in flexible bodies modelled with the ACF. The reduction mode shapes that are known from the conventional floating frame of reference based component mode synthesis [19] need to be decomposed such that they can represent the original mode in any rotated orientation. This is caused by the fact that in the original CMS method the modes are body-fixed, while in the ACF they are employed at the inertial frame, while the body can undergo arbitrary rotations. The decomposition of the original component modes is done by computing nine new generalized component modes which represent the mode related to any orientation of the body $[9,16]$. The ACF based on the new modes is also denoted as generalized component mode synthesis (GCMS), which opens a bunch of possibilities for the representation of flexible multibody systems. A beneficial characteristic of the GCMS method is its linear configuration space. That is, the displacement of any point of the flexible body is represented by a linear combination of the GCMS modes. Correspondingly, this approach can be extended to obtain a global modal parameterization (GMP) for multibody systems $[4,14]$. Furthermore, the constant mass matrix alleviates the development of energy-momentum-conserving schemes for the simulation of flexible multibody dynamics systems [11]. Finally, the extension to arbitrary Lagrange-Eulerian formulations, contact formulations, and parametric modes is facilitated because of the linear configuration space.

It is noted that the ACF is restricted to isoparametric finite elements which only contain displacement coordinates or displacement gradients. So far, finite element formulations with rotational degrees of freedom, such as beam or shell elements, do not fit into the present framework. The contribution aims at applications involving bodies that undergo finite rotations but small deformations. For a wide class of engineering applications, these bodies (a crankshaft, e.g.) are accurately modelled by means of solid finite elements with displacement degrees of freedom only. Nevertheless, it is possible to account for slender or thin geometries via the application of ANCF beam or shell elements or of so-called solid beam or solid shell elements, which are also free of rotational parameters and which fit into the present theory. Examples for ANCF beam and shell elements are given in [23,25], respectively, and for solid beam and shell elements in [5,6], respectively.

The goal of the present paper is to derive the equations of motion for the GCMS formulation in full detail to explicate its relations to the ACF and the FFRF in the first step. In a second step, the constraint conditions are formulated, which relate the GCMS to the FFRF, and it is demonstrated how the large number of rotational 
and flexible (modal) coordinates in the GCMS can be reduced by projecting them into the null-space of these constraints. This leads to an alternative and favourable approach for the derivation of the FFRF equations of motion.

The paper is organized as follows: In Sects. 2 and 3, a derivation of the equations of motion of the ACF is given. A model order reduction is applied in Sect. 4. In Sect. 5, the GCMS method is recapitulated. In Sect. 6, the constraint equations relating the ACF and the FFRF are derived. In Sect. 7, the null-space projection approach is applied to the GCMS equations of motion to finally obtain the equations of motion of the FFRF in Sect. 8. The related derivations are elementary but voluminous. A concise compilation of the fundamental results is therefore given in Sect. 8. Subsequently, a reformulation of the equations related to a local frame approach is presented. In Sect. 9, the resulting equations and corresponding solution schemes are discussed.

\section{Equations of motion}

The absolute coordinate formulation is based on a Lagrangian finite element (FE) formulation as known from textbooks on finite elements [17]. The spatial discretization of the displacement field is built upon the matrix of space-wise shape functions $\mathbf{N}^{\mathrm{FE}}(\boldsymbol{x})$ and the vector of nodal displacements $\boldsymbol{q}^{\mathrm{FE}}(t)$. The Ritz approach gives

$$
\boldsymbol{u}(\boldsymbol{x}, t)=\mathbf{N}^{\mathrm{FE}}(\boldsymbol{x}) \boldsymbol{q}^{\mathrm{FE}}(t),
$$

with $\boldsymbol{x}$ being the spatial coordinate in the reference configuration, $\boldsymbol{u}$ the displacement field, and $t$ the time. Figure 1 shows one body in the ACF, which emphasizes that the finite element mesh fully describes the motion of the deformable body.

The work of internal forces for a Saint Venant-Kirchhoff material reads

$$
W_{\text {int }}=\frac{1}{2} \int_{V} \mathbf{E}: \mathbb{C}: \mathbf{E} \mathrm{d} V,
$$

with $\mathbf{E}$ being the Green-Lagrange strain tensor and $\mathbb{C}$ the elasticity tensor.

The linear configuration space defined by (1) leads to a constant mass matrix. The equations of motion read

$$
\mathbf{M} \ddot{\boldsymbol{q}}^{\mathrm{FE}}+\frac{\partial W_{\mathrm{int}}}{\partial \boldsymbol{q}^{\mathrm{FE}}}+\left(\frac{\partial \boldsymbol{C}}{\partial \boldsymbol{q}^{\mathrm{FE}}}\right)^{T} \lambda=\boldsymbol{f}_{\mathrm{ext}}, \quad \boldsymbol{C}\left(\boldsymbol{q}^{\mathrm{FE}}\right)=\mathbf{0} .
$$

A full derivation of the equations of motion can be found in $[10,16]$.

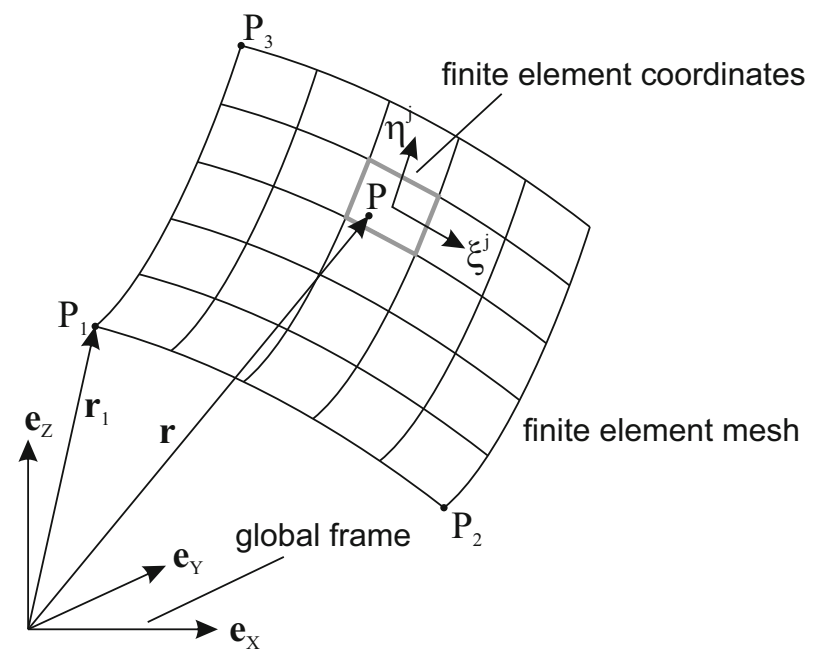

Fig. 1 Deformable bodies in the absolute coordinate formulation (ACF): the (large) motion of the body is given by the finite element mesh, while an underlying rigid body motion may be, e.g. defined by means of three points $P_{1}, P_{2}$ and $P_{3}$, using Gram-Schmidt orthonormalization. Note that the position of point $P$ is given by $\boldsymbol{r}=\boldsymbol{x}+\boldsymbol{u}$ 


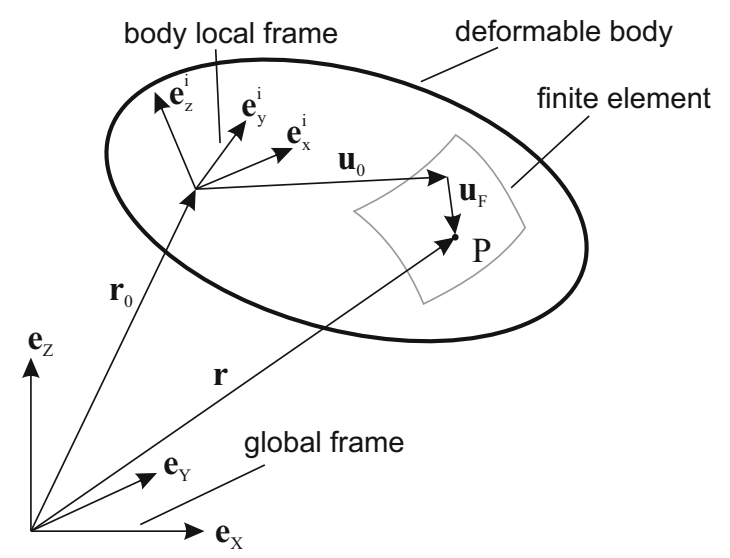

Fig. 2 In the floating frame of reference formulation, the position of a point $P$ is split into rigid body displacement $\boldsymbol{u}_{t}$, rotation $\mathbf{A} \boldsymbol{x}$ and flexible part of displacements $\boldsymbol{u}_{f}$

The mass matrix is defined as

$$
\mathbf{M}=\int_{V} \rho\left(\mathbf{N}^{\mathrm{FE}}\right)^{T} \mathbf{N}^{\mathrm{FE}} \mathrm{d} V
$$

and the vector of external forces is denoted as $f_{\text {ext }}$. Any constraints in the multibody system are given in the form

$$
\boldsymbol{C}\left(\boldsymbol{q}^{\mathrm{FE}}, t\right)=\mathbf{0},
$$

and $\lambda$ denotes the vector of Lagrange multipliers. It is noted that the equations of motion are written for a single flexible body, while in the implementation, all bodies share a large vector of coordinates. The constraints, which are applied either to the body itself (e.g. ground constraint) or which act between bodies, need to be written as a function of the coordinates of all bodies being involved in the constraints.

\section{Co-rotational formulation}

The fully nonlinear formulation of the strain energy (2) is not necessary in case of large rotations with superimposed small deformation. A convenient way to take this fact into account, is to split the displacement of a point $P$ of a single member of the multibody system in a translational $(t)$, a rotational $(r)$, and a flexible $(f)$ part, see Fig. 2,

$$
\boldsymbol{u}=\boldsymbol{u}_{t}+\underbrace{(\mathbf{A}-\mathbf{I}) \boldsymbol{x}}_{=u_{r}^{*}}+\boldsymbol{u}_{f}^{*}
$$

Therein, A represents the rotation matrix related to the rotational motion of the particular body and $\mathbf{I}$ is the $3 \times 3$ identity matrix. The rotation matrix $\mathbf{A}$ is defined by the co-rotational frame of reference $\left(\boldsymbol{d}_{1}^{*}, \boldsymbol{d}_{2}^{*}, \boldsymbol{d}_{3}^{*}\right)$ for each body. That is, $\mathbf{A}$ describes the rotation relative to the inertial frame $\left(\boldsymbol{e}_{1}, \boldsymbol{e}_{2}, \boldsymbol{e}_{3}\right)$ and it is given by

$$
\mathbf{A}=\left[\begin{array}{lll}
\boldsymbol{d}_{1}^{*} & \boldsymbol{d}_{2}^{*} & \boldsymbol{d}_{3}^{*}
\end{array}\right]
$$

The superscript ' $*$ ' has been introduced to clearly separate the role of the decomposition (6) from the one applied in the context of GCMS, see Sect. 5. In the ACF, the rotation matrix A may be retrieved in various ways, e.g. by using the position of three non-aligned points $P_{1}, P_{2}$ and $P_{3}$, see Fig. 1 , and computing the rotation matrix by means of Gram-Schmidt orthonormalization. Note that the rotation matrix can be related to the motion of the deformable body in the same way for ACF and for FRRF.

The FFRF is obtained by assigning separate coordinates to the translational, rotational and flexible part of the displacement $\boldsymbol{u}$ in Eq. (6). The decomposition of the displacement in Eq. (6) can be further refined by using the co-rotated flexible part of displacements $\overline{\boldsymbol{u}}_{f}^{*}$,

$$
\boldsymbol{u}_{f}^{*}=\mathbf{A} \overline{\boldsymbol{u}}_{f}^{*} \text {. }
$$


Due to the coupling of the components of $\mathbf{A}$, which usually have a nonlinear relation to rotational coordinates $\boldsymbol{q}_{r}^{\mathrm{FE}}$, and the flexible part of displacements $\boldsymbol{u}_{f}^{*}$, the FFRF leads to a non-constant mass matrix and a quadratic velocity vector. The computation of the inertia terms is usually time consuming, and the implementation is laborious. Related formulas involving inertia integrals can be found in [15,19,21]. An alternative approach avoiding the necessity of evaluating integrals has been given in [12], with reference to [18].

Consequently, the co-rotational strain tensor $\overline{\mathbf{E}}=\mathbf{A}^{T} \mathbf{E} \mathbf{A}$ is introduced for the FFRF and it is linearized with respect to $\overline{\boldsymbol{u}}_{f}^{*}$. This leads to the linearized strain tensor

$$
\overline{\mathbf{E}}_{\text {lin }}=\operatorname{sym}\left(\bar{\nabla} \overline{\boldsymbol{u}}_{f}^{*}\right),
$$

in which the components are represented in the body-fixed frame in case of the floating frame of reference formulation. The operator $\bar{\nabla}$ is the gradient with respect to the co-rotational coordinates $\overline{\boldsymbol{x}}=\mathbf{A}^{T} \boldsymbol{x}$.

In the ACF, the linearization of the components of the strain tensor is performed in the body frame as well,

$$
\hat{\mathbf{E}}=\operatorname{sym}\left(\mathbf{A}^{T} \nabla \boldsymbol{u}_{f}^{*}\right)=\frac{1}{2}\left[\mathbf{A}^{T} \nabla \boldsymbol{u}_{f}^{*}+\left(\nabla \boldsymbol{u}_{f}^{*}\right)^{T} \mathbf{A}\right],
$$

while all computations are applied to $\boldsymbol{u}_{f}^{*}$. It is thus necessary to relate the flexible part of deformation $\boldsymbol{u}_{f}^{*}$ from the absolute displacement field $\boldsymbol{u}(\boldsymbol{x}, t)$, which can be computed from an additive decomposition of total displacements and rigid body motion

$$
\begin{aligned}
\boldsymbol{u}_{f}^{*}(\boldsymbol{x}, t) & =\boldsymbol{u}(\boldsymbol{x}, t)-\boldsymbol{u}_{r}^{*}(\boldsymbol{x}, t)-\boldsymbol{u}_{t}(t) \\
& =\mathbf{N}^{\mathrm{FE}}(\boldsymbol{x}) \boldsymbol{q}^{\mathrm{FE}}(t)-\mathbf{N}_{r}^{\mathrm{FE}}(\boldsymbol{x}) \boldsymbol{q}_{r}^{\mathrm{FE}}(t)-\boldsymbol{q}_{t}^{\mathrm{FE}}(t) \\
& =\mathbf{N}_{f}^{\mathrm{FE}}(\boldsymbol{x}) \boldsymbol{q}_{f}^{\mathrm{FE}}(t),
\end{aligned}
$$

noting that the work of elastic forces is zero for rigid body displacements $\boldsymbol{u}_{r}^{*}+\boldsymbol{u}_{t}$ and that $\boldsymbol{q}_{r}^{\mathrm{FE}}$ and $\boldsymbol{q}_{f}^{\mathrm{FE}}$ are implicitly defined by Eq. (11) and finally that $\boldsymbol{q}_{t}^{\mathrm{FE}}=\boldsymbol{u}_{t}$. Thus, the variation of the strain energy can be written as

$$
\begin{aligned}
\delta W_{\mathrm{int}} & =\int_{V}\left(\left(\nabla \boldsymbol{u}_{f}^{*}\right)^{T} \mathbf{A}\right): \mathbb{C}:\left(\mathbf{A}^{T} \delta \nabla \boldsymbol{u}_{f}^{*}+\delta \mathbf{A}^{T} \nabla \boldsymbol{u}_{f}^{*}\right) \mathrm{d} V \\
& =\left(\boldsymbol{q}_{f}^{\mathrm{FE}}\right)^{T} \mathbf{A}_{\mathrm{bd}} \mathbf{K} \mathbf{A}_{\mathrm{bd}}^{T} \delta \boldsymbol{q}^{\mathrm{FE}}+\delta \boldsymbol{f}_{\mathrm{nl}}^{T}\left(\boldsymbol{q}^{\mathrm{FE}}\right),
\end{aligned}
$$

in which $\mathbf{K}$ represents the constant (co-rotational) stiffness matrix and $\boldsymbol{f}_{\mathrm{nl}}$ is a small nonlinear term, which depends quadratically on $\boldsymbol{q}_{f}^{\mathrm{FE}}$. At this point, it is required that $\boldsymbol{q}^{\mathrm{FE}}$ represents the displacement components of each FE node alternately for the $x, y$ and $z$ components, i.e.

$$
\boldsymbol{q}^{\mathrm{FE}}=\left[\begin{array}{lllllll}
q_{x 1}^{\mathrm{FE}} & q_{y 1}^{\mathrm{FE}} & q_{z 1}^{\mathrm{FE}} & q_{x 2}^{\mathrm{FE}} & q_{y 2}^{\mathrm{FE}} & q_{z 2}^{\mathrm{FE}} & \ldots
\end{array}\right]^{T} .
$$

Therefore, the rotation of the stiffness matrix can be written by means of a block diagonal matrix $\mathbf{A}_{\text {bd }}$, which contains the rotation matrices $\mathbf{A}$ at its diagonal, see [9] for details.

\section{Modal reduction}

In flexible multibody dynamics, the deformation of every body is usually modelled by means of finite elements. In many applications, there is only a small number of important deformation modes in each body. Therefore, the so-called component mode synthesis (CMS) method has been developed, which is based on a small number of static and dynamic mode shapes that accurately describe the deformation of the bodies.

The flexible part of displacement $\overline{\boldsymbol{u}}_{f}$, given in body-fixed coordinates, is thus approximated by

$$
\overline{\boldsymbol{u}}_{f}^{*}(\boldsymbol{x}, t) \approx \sum_{\mu=1}^{M} \mathbf{N}_{f, \mu}(\boldsymbol{x}) q_{f, \mu}^{\prime}(t),
$$


with $M$ being the number of flexible modes chosen, which is usually much smaller than the number of nodal coordinates of the underlying body's finite element mesh. The reduced flexible coordinates $q_{f, \mu}^{\prime}$ introduced in (14) coincide with the ones of the FFRF. The modes are given by

$$
\mathbf{N}_{f, \mu}(\boldsymbol{x})=\mathbf{N}^{\mathrm{FE}}(\boldsymbol{x}) \boldsymbol{\phi}_{f, \mu},
$$

where $\boldsymbol{\phi}_{f, \mu}$ is the vector containing the nodal displacements related to the $\mu$ th component mode. In principle, the set of component modes may subsume static and dynamic ones. Here, we only consider eigenmodes, such that $\boldsymbol{\phi}_{f, \mu}$ is related to an eigenfrequency $\omega_{\mu}$ and follows from

$$
\left(\mathbf{K}-\omega_{\mu}^{2} \mathbf{M}\right) \boldsymbol{\phi}_{f, \mu}=\mathbf{0} .
$$

In total, the reduced kinematics of a flexible body are represented by three translational, three rotational, and $M$ flexible coordinates.

In the ACF, the modes need to be transformed into absolute or inertial coordinates, using the rotation matrix A. Thus, the flexible part of the displacement vector is given as

$$
\boldsymbol{u}_{f}^{*}=\mathbf{A} \overline{\boldsymbol{u}}_{f}^{*}=\sum_{\mu=1}^{M} \sum_{i, j=1}^{3} \boldsymbol{e}_{j} A_{j i} N_{f, \mu i} q_{f, \mu}^{\prime},
$$

where $A_{j i}=\boldsymbol{e}_{j} \cdot \boldsymbol{d}_{i}^{*}$ and $N_{f, \mu i}=\boldsymbol{e}_{i} \cdot \mathbf{N}_{f, \mu}$. Note that there is a nonlinear dependence of $\boldsymbol{u}_{f}$ on the generalized coordinates. The search for an alternative decomposition of $\boldsymbol{u}_{f}$ which preserves the linear nature of the Ritz approach (1) leads to the GCMS method described in the next section.

\section{Generalized component mode synthesis (GCMS)}

The general idea of generalized component modes [9] is to maintain a linear configuration space for the translational, rotational, and flexible part of displacements. That is,

$$
\boldsymbol{u}(\boldsymbol{x})=\mathrm{N}^{\mathrm{GCMS}}(\boldsymbol{x}) \boldsymbol{q},
$$

in which the matrix $\mathbf{N}^{\mathrm{GCMS}}$ has size $3 \times N_{\mathrm{GCMS}}$ and contains the mode shapes according to the generalized coordinates

$$
\boldsymbol{q}=\left[\begin{array}{lll}
\boldsymbol{q}_{t}^{T} & \boldsymbol{q}_{r}^{T} & \boldsymbol{q}_{f}^{T}
\end{array}\right]^{T} .
$$

In general, the spatial movement of a deformable body is modelled by means of three mode shapes for translation, nine mode shapes for rotation and $9 M$ mode shapes for the flexible part of displacements, based on $M$ static or dynamic mode shapes. The total number of generalized modes for the GCMS is thus $N_{\mathrm{GCMS}}=$ $12+9 M$. Note that the nine mode shapes which represent the rotational motion also include deformation.

\subsection{Representation of rigid body motion}

The idea to represent rigid body motion by means of twelve coordinates is well known, see e.g. [3,7]. The translational and rotational parts of the displacement vector can be written as

$$
\begin{aligned}
& \boldsymbol{u}_{t}=\sum_{i=1}^{3} u_{t, i} \boldsymbol{e}_{i}=\sum_{i=1}^{3} q_{t, i} \mathbf{N}_{t, i}^{\mathrm{GCMS}}, \\
& \boldsymbol{u}_{r}^{*}=(\mathbf{A}-\mathbf{I}) \boldsymbol{x}=\sum_{i, j=1}^{3} q_{r, i j}^{*} \mathbf{N}_{r, i j}^{\mathrm{GCMS}}(\boldsymbol{x}),
\end{aligned}
$$

in which the generalized coordinates are related to rigid body translation $\boldsymbol{u}_{t}$ and the rotation matrix $\mathbf{A}$ in the following way:

$$
q_{t, i}=u_{t, i},
$$




$$
q_{r, i j}^{*}=A_{j i}-\delta_{i j}
$$

together with shape functions

$$
\begin{aligned}
& \mathbf{N}_{t, i}^{\mathrm{GCMS}}(\boldsymbol{x}) \equiv \boldsymbol{e}_{i}, \\
& \mathbf{N}_{r, i j}^{\mathrm{GCMS}}(\boldsymbol{x}) \equiv x_{i} \boldsymbol{e}_{j} .
\end{aligned}
$$

According to their definition (23), the nine coordinates $q_{r, i j}^{*}$ refer to a rigid body rotation and are thus subjected to six constraint equations. It is important to notice that the related shape functions (25) subsume the three rotational modes together with six additional deformational modes. In the context of the GCMS formulation, the nine degrees of freedom corresponding to these nine modes are employed as rotation-type coordinates and are denoted as $q_{r, i j}$. These are handled to be independent of each other and thus represent, primarily, the rigid body rotation and, in addition, a deformational part $\varepsilon_{r, i j}$. That is,

$$
q_{r, i j}=q_{r, i j}^{*}+\varepsilon_{r, i j}
$$

This gives rise to a redefinition of the rotation-type part of the absolute displacement field,

$$
\boldsymbol{u}_{r}=\sum_{i, j=1}^{3} q_{r, i j} \mathbf{N}_{r, i j}^{\mathrm{GCMS}}(\boldsymbol{x}) .
$$

The interrelation of finite element and modal spaces is given in analogy to (15),

$$
\begin{aligned}
& \mathbf{N}_{t, i}^{\mathrm{GCMS}}(\boldsymbol{x})=\mathbf{N}^{\mathrm{FE}}(\boldsymbol{x}) \boldsymbol{\phi}_{t, i}=\underbrace{\sum_{n=1}^{N} N_{n}^{\mathrm{FE}}(\boldsymbol{x}) \boldsymbol{e}_{i},}_{=1} \\
& \mathbf{N}_{r, i j}^{\mathrm{GCMS}}(\boldsymbol{x})=\mathbf{N}^{\mathrm{FE}}(\boldsymbol{x}) \boldsymbol{\phi}_{r, i j}(\boldsymbol{x})=\underbrace{\sum_{n=1}^{N} N_{n}^{\mathrm{FE}}(\boldsymbol{x}) x_{n i}}_{=x_{i}} \boldsymbol{e}_{j} .
\end{aligned}
$$

Therein, the vectors

$$
\begin{aligned}
\boldsymbol{\phi}_{t, j} & =\left[\begin{array}{llll}
\underbrace{\boldsymbol{e}_{j}^{T}}_{N \text { times }} \boldsymbol{e}_{j}^{T} & \cdots & \boldsymbol{e}_{j}^{T}
\end{array}\right]^{T} \\
\boldsymbol{\phi}_{r, i j} & =\left[\begin{array}{llll}
x_{1 i} \boldsymbol{e}_{j}^{T} & x_{2 i} \boldsymbol{e}_{j}^{T} & \cdots & x_{N i} \boldsymbol{e}_{j}^{T}
\end{array}\right]^{T}
\end{aligned}
$$

are the displacement modes representing translational and rotational motion, respectively. Their matrix dimensions are $3 N \times 1$, with $N$ being the number of finite element nodes. Further, $\boldsymbol{x}_{n}=\left[\begin{array}{lll}x_{n 1} & x_{n 2} & x_{n 3}\end{array}\right]^{T}$ is the position vector of finite element node $n=1, \ldots, N$ and $N_{n}^{\mathrm{FE}}$ is the corresponding FE shape function with the standard properties

$$
N_{n}^{\mathrm{FE}}\left(\boldsymbol{x}_{m}\right)=\delta_{n m}, \quad \sum_{n=1}^{N} N_{n}^{\mathrm{FE}}(\boldsymbol{x}) \equiv 1
$$

and

$$
\mathbf{N}^{\mathrm{FE}}(\boldsymbol{x})=\left[\begin{array}{lll}
N_{1}^{\mathrm{FE}}(\boldsymbol{x}) \mathbf{I} & \cdots & N_{n}^{\mathrm{FE}}(\boldsymbol{x}) \mathbf{I}
\end{array}\right] .
$$

Introducing the modal reduction matrices

$$
\boldsymbol{\Phi}_{t}=\left[\begin{array}{lll}
\boldsymbol{\phi}_{t, 1} & \boldsymbol{\phi}_{t, 2} & \boldsymbol{\phi}_{t, 3}
\end{array}\right]=\left[\begin{array}{c}
\mathbf{I} \\
\vdots \\
\mathbf{I}
\end{array}\right]
$$


and

$$
\boldsymbol{\Phi}_{r}=\left[\begin{array}{llllll}
\boldsymbol{\phi}_{r, 11} & \boldsymbol{\phi}_{r, 12} & \boldsymbol{\phi}_{r, 13} & \boldsymbol{\phi}_{r, 21} & \cdots & \boldsymbol{\phi}_{r, 33}
\end{array}\right]=\left[\begin{array}{ccc}
x_{1}^{1} \mathbf{I} & x_{1}^{2} \mathbf{I} & x_{1}^{3} \mathbf{I} \\
\vdots & \vdots & \vdots \\
x_{N}^{1} \mathbf{I} & x_{N}^{2} \mathbf{I} & x_{N}^{3} \mathbf{I}
\end{array}\right]
$$

of dimensions $3 N \times 3$ and $3 N \times 9$, respectively, one has

$$
\mathbf{N}_{t}^{\mathrm{GCMS}}=\mathbf{N}^{\mathrm{FE}} \boldsymbol{\Phi}_{t}, \quad \mathbf{N}_{r}^{\mathrm{GCMS}}=\mathbf{N}^{\mathrm{FE}} \boldsymbol{\Phi}_{r} .
$$

In view of computer implementations, the rigid body coordinates (22) and (26) are organized as vectors

$$
\boldsymbol{q}_{t}=\boldsymbol{u}_{t}, \quad \boldsymbol{q}_{r}=\left[\begin{array}{ccc}
\boldsymbol{q}_{r 1}^{T} & \boldsymbol{q}_{r 2}^{T} & \boldsymbol{q}_{r 3}^{T}
\end{array}\right]^{T}
$$

of dimensions $3 \times 1$ and $9 \times 1$, respectively. Thus, Eqs. (20) and (27) can be written as

$$
\boldsymbol{u}_{t}=\mathbf{N}_{t}^{\mathrm{GCMS}} \boldsymbol{q}_{t}, \quad \boldsymbol{u}_{r}=\mathbf{N}_{r}^{\mathrm{GCMS}} \boldsymbol{q}_{r} .
$$

The $\boldsymbol{q}_{r i}$ are the column vectors of the $3 \times 3$ matrix

$$
\mathbf{Q}_{r}^{T}=\left[\begin{array}{lll}
\boldsymbol{q}_{r 1} & \boldsymbol{q}_{r 2} & \boldsymbol{q}_{r 3}
\end{array}\right] .
$$

In the case of pure rigid body motion, $\mathbf{Q}_{r}^{T}=\mathbf{A}-\mathbf{I}$ and $\boldsymbol{q}_{r i}=\boldsymbol{d}_{i}^{*}-\boldsymbol{e}_{i}$. This gives rise to the definition

$$
\boldsymbol{d}_{i}=\boldsymbol{q}_{r i}+\boldsymbol{e}_{i}, \quad i=1,2,3 .
$$

Note that, in general, $\boldsymbol{d}_{i} \neq \boldsymbol{d}_{i}^{*}$.

\subsection{Representation of deformation}

The flexible part $\boldsymbol{u}_{f}^{*}$ of the displacement vector has been defined in (6). The decomposition of $\boldsymbol{u}_{f}^{*}$ introduced in (17) gives rise to the definition of the variables

$$
q_{f, \mu i j}^{*}=A_{j i} q_{f, \mu}^{\prime}, \quad \mu=1, \ldots, M \text { and } i, j=1,2,3
$$

replacing the co-rotational flexible degrees of freedom $q_{f, \mu}^{\prime}$. Each variable $q_{f, \mu i j}^{*}$ is related to a corresponding, so-called generalized component mode,

$$
\mathbf{N}_{f, \mu i j}^{\mathrm{GCMS}}=N_{f, \mu i} \boldsymbol{e}_{j}
$$

For a given orthogonal matrix $A_{i j}$ and for each mode $\mu$, the nine $q_{f, \mu i j}^{*}(i, j=1,2,3)$ are subjected to eight constraint conditions. In contrast, in the context of the GCMS technique, the $9 M$ variables related to the $9 M$ generalized component modes are considered to constitute a set of independent coordinates $q_{f, \mu i j}$. They account for deformational and rotational motion. This is also the case for the corresponding part of the deformation vector,

$$
\boldsymbol{u}_{f}=\sum_{\mu=1}^{M} \sum_{i, j=1}^{3} \mathbf{N}_{f, \mu i j}^{\mathrm{GCMS}} q_{f, \mu i j}=\mathbf{N}_{f}^{\mathrm{GCMS}} \boldsymbol{q}_{f} .
$$

The term 'generalized' should indicate that the original modes are generalized (or enhanced) such that they can represent a mode in any rotated configuration. For each mode $\mu=1, \ldots, M$, the nine flexible coordinates $q_{f, \mu i j}$ can be arranged to constitute a matrix

$$
\mathbf{Q}_{f, \mu}^{T}=\left[\begin{array}{lll}
\boldsymbol{q}_{\mu 1} & \boldsymbol{q}_{\mu 2} & \boldsymbol{q}_{\mu 3}
\end{array}\right]
$$

consisting of the three column vectors

$$
\boldsymbol{q}_{\mu j}=\left[\begin{array}{lll}
q_{\mu 1 j} & q_{\mu 2 j} & q_{\mu 3 j}
\end{array}\right]^{T}, \quad j=1,2,3 .
$$

In the case of pure rigid body motion, one has

$$
\boldsymbol{q}_{\mu j}=q_{f, \mu}^{\prime} \boldsymbol{d}_{j}^{*}
$$


and thus

$$
\mathbf{Q}_{f, \mu}^{T}=q_{f, \mu}^{\prime} \mathbf{A} \text {. }
$$

Note that, in general, (47) does not hold. With respect to computer implementation, it is more favourable to arrange the flexible coordinates as a vector of dimensions $3 M \times 1$,

$$
\boldsymbol{q}_{f}=\left[\begin{array}{lllllll}
\boldsymbol{q}_{11}^{T} & \boldsymbol{q}_{12}^{T} & \boldsymbol{q}_{13}^{T} & \cdots & \boldsymbol{q}_{M 1}^{T} & \boldsymbol{q}_{M 2}^{T} & \boldsymbol{q}_{M 3}^{T}
\end{array}\right]^{T} .
$$

Inserting (15) into (42), the GCMS shape functions related to the flexible part become

$$
N_{f, \mu i j}^{\mathrm{GCMS}}(\boldsymbol{x})=\mathbf{N}^{\mathrm{FE}}(\boldsymbol{x}) \boldsymbol{\phi}_{f, \mu i j}
$$

with the $3 N \times 1$ vectors

$$
\boldsymbol{\phi}_{f, \mu i j}=\left[\begin{array}{llll}
\phi_{f, \mu i} \boldsymbol{e}_{j}^{T} & \phi_{f, \mu(3+i)} \boldsymbol{e}_{j}^{T} & \cdots & \phi_{f, \mu(3(N-1)+i)} \boldsymbol{e}_{j}^{T}
\end{array}\right]^{T}
$$

for $\mu=1, \ldots, M$ and $i, j=1,2,3$. The $\phi_{f, \mu k}$ denotes the $k$ th component of the flexible mode vector $\phi_{f, \mu}$. It is convenient to introduce an alternative notation:

$$
\phi_{f, \mu}^{i}\left(\boldsymbol{x}_{n}\right)=\phi_{f, \mu(3(n-1)+i)}
$$

shall denote the $i$ th component of the amplitude of flexible mode number $\mu$ at finite element node number $n$. Compiling the column vectors $\boldsymbol{\phi}_{f, \mu i j}$, a $3 N \times 9$ matrix is obtained,

$$
\begin{aligned}
& \boldsymbol{\Phi}_{f, \mu}=\left[\begin{array}{lllllll}
\boldsymbol{\phi}_{f, \mu 11} & \boldsymbol{\phi}_{f, \mu 12} & \boldsymbol{\phi}_{f, \mu 13} & \boldsymbol{\phi}_{f, \mu 21} & \cdots & \boldsymbol{\phi}_{f, \mu 33}
\end{array}\right] \\
& =\left[\begin{array}{ccc}
\phi_{f, \mu}^{1}\left(\boldsymbol{x}_{1}\right) \mathbf{I} & \phi_{f, \mu}^{2}\left(\boldsymbol{x}_{1}\right) \mathbf{I} & \phi_{f, \mu}^{3}\left(\boldsymbol{x}_{1}\right) \mathbf{I} \\
\vdots & \vdots & \vdots \\
\phi_{f, \mu}^{1}\left(\boldsymbol{x}_{N}\right) \mathbf{I} & \phi_{f, \mu}^{2}\left(\boldsymbol{x}_{N}\right) \mathbf{I} & \phi_{f, \mu}^{3}\left(\boldsymbol{x}_{N}\right) \mathbf{I}
\end{array}\right],
\end{aligned}
$$

and further the $3 N \times 9 M$ matrix

$$
\boldsymbol{\Phi}_{f}=\left[\begin{array}{llll}
\boldsymbol{\Phi}_{f, 1} & \boldsymbol{\Phi}_{f, 2} & \cdots & \boldsymbol{\Phi}_{f, M}
\end{array}\right] .
$$

Accordingly, the shape functions (49) are subsumed to become

$$
\mathbf{N}_{f}^{\mathrm{GCMS}}(\boldsymbol{x})=\mathbf{N}^{\mathrm{FE}}(\boldsymbol{x}) \boldsymbol{\Phi}_{f} .
$$

\subsection{Reduced equations of motion}

Introducing the GCMS reduction matrix

$$
\boldsymbol{\Phi}^{\mathrm{GCMS}}=\left[\begin{array}{lll}
\boldsymbol{\Phi}_{t} & \boldsymbol{\Phi}_{r} & \boldsymbol{\Phi}_{f}
\end{array}\right]
$$

and defining the corresponding mass and stiffness matrices

$$
\begin{gathered}
\overline{\mathbf{M}}=\left(\boldsymbol{\Phi}^{\mathrm{GCMS}}\right)^{T} \mathbf{M} \boldsymbol{\Phi}^{\mathrm{GCMS}}, \\
\overline{\mathbf{K}}=\left(\boldsymbol{\Phi}^{\mathrm{GCMS}}\right)^{T} \mathbf{K} \boldsymbol{\Phi}^{\mathrm{GCMS}},
\end{gathered}
$$

respectively, and the force vector

$$
\overline{\boldsymbol{f}}_{\mathrm{ext}}=\left(\boldsymbol{\Phi}^{\mathrm{GCMS}}\right)^{T} \boldsymbol{f}_{\mathrm{ext}},
$$

the reduced equations of motions become

$$
\overline{\mathbf{M}} \ddot{\boldsymbol{q}}+\mathbf{A}_{\mathrm{bd}} \overline{\mathbf{K}} \mathbf{A}_{\mathrm{bd}}^{T} \boldsymbol{q}_{\mathrm{flex}}+\overline{\boldsymbol{f}}_{\mathrm{nl}}+\overline{\mathbf{G}}^{T} \boldsymbol{\lambda}=\overline{\boldsymbol{f}}_{\mathrm{ext}} .
$$

Therein, $\mathbf{A}_{\mathrm{bd}}$ again denotes an appropriate block diagonal matrix applying the rigid body rotation $\mathbf{A}$ to each $3 \times 1$ block of the coordinate vector $\boldsymbol{q}$. Note that $\mathbf{A}_{\mathrm{bd}}$ and $\boldsymbol{\Phi}^{\mathrm{GCMS}}$ commute [16]! As mentioned above, in the original GCMS formulation the displacement related to the $\boldsymbol{q}_{r}$ coordinates is not purely rotational. That 
is, the matrix $\mathbf{I}+\mathbf{Q}_{r}$ referring to the rotational degrees of freedom is not necessarily orthogonal. Applying an appropriate orthogonalization procedure, proper rotational coordinates $\boldsymbol{q}_{r}^{*}$ can be defined [16]. Thus, the flexible coordinates become

$$
\boldsymbol{q}_{\mathrm{flex}}=\left[\begin{array}{lll}
\mathbf{0}^{T} & \left(\boldsymbol{q}_{r}-\boldsymbol{q}_{r}^{*}\right)^{T} & \boldsymbol{q}_{f}^{T}
\end{array}\right]^{T} .
$$

The nonlinear force vector $\overline{\boldsymbol{f}}_{\mathrm{nl}}$ is given by

$$
\overline{\boldsymbol{f}}_{\mathrm{nl}}^{T}=\sum_{i, j=1}^{3} \underbrace{\boldsymbol{q}_{\mathrm{flex}}^{T} \mathbf{A}_{\mathrm{bd}} \overline{\mathbf{K}} \frac{\partial \mathbf{A}_{\mathrm{bd}}^{T}}{\partial A_{i j}} \boldsymbol{q}_{\text {flex }}}_{\text {scalar }} \frac{\partial A_{i j}}{\partial \boldsymbol{q}^{\mathrm{GCMS}}} .
$$

Due to the sparse structure of the partial derivatives of the rotation matrices, the computation of $\overline{\boldsymbol{f}}_{\mathrm{nl}}$ can be implemented in a very efficient way. It will turn out later that the nonlinear forces vanish in the course of the null-space projection. The constraint Jacobian matrix $\overline{\mathbf{G}}=\mathbf{G} \boldsymbol{\Phi}^{\mathrm{GCMS}}$ will be dealt with in the next section.

\section{Constraint conditions}

In this section, possible constraint conditions are considered. The vector equation

$$
\boldsymbol{C}_{b}(\boldsymbol{q})=\mathbf{0}
$$

refers to single- or multibody constraints such as support or joint conditions. Constraint equations specifically related to the GCMS formulation will be denoted as $\boldsymbol{C}_{r}(\boldsymbol{q})=\mathbf{0}$ and $\boldsymbol{C}_{f}(\boldsymbol{q})=\mathbf{0}$. Constraints $\boldsymbol{C}_{r}$ enforce the rotational coordinates $\boldsymbol{q}_{r}$ to refer to a proper rigid body motion according to (39). The constraints $\boldsymbol{C}_{f}$ impose the condition (47) on the flexible coordinates $\boldsymbol{q}_{f}$. In this work, conditions $\boldsymbol{C}_{b}$ are implemented by means of the Lagrangian multiplier method, whereas a null-space projection approach is applied for $\boldsymbol{C}_{r}$ and $\boldsymbol{C}_{f}$. Originally, null-space methods have emerged in the context of optimization theory. In view of mechanical applications, a related method has been introduced in [13] and further elaborated in [2,3], e.g.

The corresponding constraint Jacobian matrices are denoted by

$$
\begin{aligned}
& \mathbf{C}_{r t}=\frac{\partial \boldsymbol{C}_{r}}{\partial \boldsymbol{q}_{t}}, \quad \mathbf{C}_{r r}=\frac{\partial \boldsymbol{C}_{r}}{\partial \boldsymbol{q}_{r}}, \quad \mathbf{C}_{r} f=\frac{\partial \boldsymbol{C}_{r}}{\partial \boldsymbol{q}_{f}}, \\
& \mathbf{C}_{f t}=\frac{\partial \boldsymbol{C}_{f}}{\partial \boldsymbol{q}_{t}}, \quad \mathbf{C}_{f r}=\frac{\partial \boldsymbol{C}_{f}}{\partial \boldsymbol{q}_{r}}, \quad \mathbf{C}_{f f}=\frac{\partial \boldsymbol{C}_{f}}{\partial \boldsymbol{q}_{f}}, \\
& \mathbf{C}_{b t}=\frac{\partial \boldsymbol{C}_{b}}{\partial \boldsymbol{q}_{t}}, \quad \mathbf{C}_{b r}=\frac{\partial \boldsymbol{C}_{b}}{\partial \boldsymbol{q}_{r}}, \quad \mathbf{C}_{b f}=\frac{\partial \boldsymbol{C}_{b}}{\partial \boldsymbol{q}_{f}} .
\end{aligned}
$$

\subsection{Constraining the rigid body coordinates}

Here, a point of view is taken opposite to the one in Sect. 5.1. That is, the directors of the body frame $\left(\boldsymbol{d}_{1}, \boldsymbol{d}_{2}, \boldsymbol{d}_{3}\right)$ are now defined based on the a priori independent coordinates $\boldsymbol{q}_{r i}$,

$$
\boldsymbol{d}_{i}:=\boldsymbol{q}_{r i}+\boldsymbol{e}_{i}
$$

Note that there is a conceptual difference between the $\boldsymbol{d}_{i}$ and the $\boldsymbol{d}_{i}^{*}$ introduced in Sect. 3. The conditions enforcing the orthonormality of the triad $\left(\boldsymbol{d}_{1}, \boldsymbol{d}_{2}, \boldsymbol{d}_{3}\right)$ are

$$
\boldsymbol{C}_{r}\left(\boldsymbol{q}_{r}\right)=\left[\begin{array}{c}
\frac{1}{2}\left(\boldsymbol{d}_{1}^{T} \boldsymbol{d}_{1}-1\right) \\
\frac{1}{2}\left(\boldsymbol{d}_{2}^{T} \boldsymbol{d}_{2}-1\right) \\
\frac{1}{2}\left(\boldsymbol{d}_{3}^{T} \boldsymbol{d}_{3}-1\right) \\
\boldsymbol{d}_{1}^{T} \boldsymbol{d}_{2} \\
\boldsymbol{d}_{1}^{T} \boldsymbol{d}_{3} \\
\boldsymbol{d}_{2}^{T} \boldsymbol{d}_{3}
\end{array}\right]=\mathbf{0} .
$$


According to (63), the relevant block of the constraint Jacobian reads

$$
\mathbf{C}_{r r}^{(6 \times 9)}=\left[\begin{array}{lll}
\boldsymbol{d}_{1}^{T} & \mathbf{0}^{T} & \boldsymbol{0}^{T} \\
\mathbf{0}^{T} & \boldsymbol{d}_{2}^{T} & \boldsymbol{0}^{T} \\
\mathbf{0}^{T} & \mathbf{0}^{T} & \boldsymbol{d}_{3}^{T} \\
\boldsymbol{d}_{2}^{T} & \boldsymbol{d}_{1}^{T} & \mathbf{0}^{T} \\
\boldsymbol{d}_{3}^{T} & \mathbf{0}^{T} & \boldsymbol{d}_{1}^{T} \\
\mathbf{0}^{T} & \boldsymbol{d}_{3}^{T} & \boldsymbol{d}_{2}^{T}
\end{array}\right]
$$

whereas

$$
\mathbf{C}_{r t}=\mathbf{O}^{(6 \times 3)}, \quad \mathbf{C}_{r} f=\mathbf{O}^{(6 \times 9 M)} .
$$

Note that the italic letter $\boldsymbol{C}$ is used to denote a vector of constraint expressions, whereas the upright letter $\mathbf{C}$ refers to the corresponding Jacobian matrix.

\subsection{Constraining the flexible coordinates}

The condition (47) can be reformulated to become

$$
\mathbf{A}^{T} \mathbf{Q}_{f, \mu}=q_{f, \mu}^{\prime} \mathbf{I}
$$

A necessary condition for (68) is

$$
\boldsymbol{C}_{f, \mu}(\boldsymbol{q})=\left[\begin{array}{c}
\boldsymbol{d}_{2}^{T} \boldsymbol{q}_{\mu 1} \\
\boldsymbol{d}_{3}^{T} \boldsymbol{q}_{\mu 1} \\
\boldsymbol{d}_{1}^{T} \boldsymbol{q}_{\mu 2} \\
\boldsymbol{d}_{3}^{T} \boldsymbol{q}_{\mu 2} \\
\boldsymbol{d}_{1}^{T} \boldsymbol{q}_{\mu 3} \\
\boldsymbol{d}_{2}^{T} \boldsymbol{q}_{\mu 3} \\
\boldsymbol{d}_{1}^{T} \boldsymbol{q}_{\mu 1}-\boldsymbol{d}_{2}^{T} \boldsymbol{q}_{\mu 2} \\
\boldsymbol{d}_{1}^{T} \boldsymbol{q}_{\mu 1}-\boldsymbol{d}_{3}^{T} \boldsymbol{q}_{\mu 3}
\end{array}\right]=\mathbf{0}
$$

Note that the first six lines correspond to the off-diagonal terms of $\mathbf{A}^{T} \mathbf{Q}_{f, \mu}$, while the last two enforce the pairwise equality of the diagonal terms. Equation (69) constitutes a feasible set of constraint equations referring to the $\mu$ th flexible mode. The respective constraint Jacobians read

$$
\mathbf{C}_{f f, \mu}^{(8 \times 9)}=\left[\begin{array}{ccc}
\boldsymbol{d}_{2}^{T} & \mathbf{0}^{T} & \mathbf{0}^{T} \\
\boldsymbol{d}_{3}^{T} & \mathbf{0}^{T} & \mathbf{0}^{T} \\
\mathbf{0}^{T} & \boldsymbol{d}_{1}^{T} & \mathbf{0}^{T} \\
\mathbf{0}^{T} & \boldsymbol{d}_{3}^{T} & \mathbf{0}^{T} \\
\mathbf{0}^{T} & \mathbf{0}^{T} & \boldsymbol{d}_{1}^{T} \\
\mathbf{0}^{T} & \mathbf{0}^{T} & \boldsymbol{d}_{2}^{T} \\
\boldsymbol{d}_{1}^{T} & -\boldsymbol{d}_{2}^{T} & \mathbf{0}^{T} \\
\boldsymbol{d}_{1}^{T} & \mathbf{0}^{T} & -\boldsymbol{d}_{3}^{T}
\end{array}\right], \quad \mathbf{C}_{f r, \mu}^{(8 \times 9)}=\left[\begin{array}{ccc}
\mathbf{0}^{T} & \boldsymbol{q}_{\mu 1}^{T} & \mathbf{0}^{T} \\
\mathbf{0}^{T} & \mathbf{0}^{T} & \boldsymbol{q}_{\mu 1}^{T} \\
\boldsymbol{q}_{\mu 2}^{T} & \mathbf{0}^{T} & \mathbf{0}^{T} \\
\mathbf{0}^{T} & \mathbf{0}^{T} & \boldsymbol{q}_{\mu 2}^{T} \\
\boldsymbol{q}_{\mu 3}^{T} & \mathbf{0}^{T} & \mathbf{0}^{T} \\
\mathbf{0}^{T} & \boldsymbol{q}_{\mu 3}^{T} & \mathbf{0}^{T} \\
\boldsymbol{q}_{\mu 1}^{T} & -\boldsymbol{q}_{\mu 2}^{T} & \mathbf{0}^{T} \\
\boldsymbol{q}_{\mu 1}^{T} & \mathbf{0}^{T} & -\boldsymbol{q}_{\mu 3}^{T}
\end{array}\right]
$$

and

$$
\mathbf{C}_{f t}=\mathbf{O}^{(8 \times 3)} \text {. }
$$




\subsection{Null-space projection}

The null-space (or kernel) $\mathcal{K}$ of a $l \times m$ matrix $\mathbf{C} \in \mathbb{R}^{l \times m}$ is the set of all $m$ vectors $\boldsymbol{v} \in \mathbb{R}^{m}$ for which $\mathbf{C} \boldsymbol{v}=\mathbf{0} \in \mathbb{R}^{l}$. Obviously, the null-space is a vector space and a subspace of $\mathbb{R}^{m}$. Correspondingly, a null-space projection (matrix) is a matrix $\mathbf{P} \in \mathbb{R}^{m \times n}$ which maps all vectors of $\mathbb{R}^{n}$ on the null-space of $\mathbf{C}$. If $\mathbf{P}$ has rank $n$, i.e. $\mathbf{P}$ has $n$ linearly independent row (or, equivalently, column) vectors, the mapping $\mathbf{P}$ is onto, i.e. for every $\boldsymbol{w} \in \mathcal{K}$ there is a $\boldsymbol{v} \in \mathbb{R}^{m}$ such that $\boldsymbol{w}=\mathbf{P} \boldsymbol{v}$. This is possible only if $n \leq m$. Then, $\operatorname{rank}(\mathbf{P})=n$ means that $\mathbf{P}$ has full rank.

For the present purpose, the relevant constraint conditions are $\boldsymbol{C}_{r}$ and $\boldsymbol{C}_{f}$. The corresponding constraint Jacobian reads

$$
\mathbf{C}=\left[\begin{array}{lll}
\mathbf{O}^{(6 \times 3)} & \mathbf{C}_{r r}^{(6 \times 9)} & \mathbf{O}^{(6 \times 9 M)} \\
\mathbf{O}^{(8 \times 3)} & \mathbf{C}_{f r}^{(8 \times 9)} & \mathbf{C}_{f f}^{(8 \times 9 M)}
\end{array}\right] .
$$

A proper projection matrix has the shape

$$
\mathbf{P}=\left[\begin{array}{ccc}
\mathbf{I}^{(3 \times 3)} & \mathbf{O}^{(3 \times 3)} & \mathbf{O}^{(3 \times M)} \\
\mathbf{O}^{(9 \times 3)} & \mathbf{P}_{r r}^{(9 \times 3)} & \mathbf{O}^{(9 \times M)} \\
\mathbf{O}^{(9 M \times 3)} & \mathbf{P}_{f r}^{(9 M \times 3)} & \mathbf{P}_{f f}^{(9 M \times M)}
\end{array}\right]
$$

The first three columns of $\mathbf{C}$ and $\mathbf{P}$ and the three rows of $\mathbf{P}$ refer to the translational coordinates which are not subjected to rigid body constraints and are thus not projected. The characterizing properties of $\mathbf{P}$ are

$$
\mathbf{C P}=\mathbf{O}^{[(6+8) \times(3+3+M)]} \text { and } \operatorname{rank}(\mathbf{P})=3+3+M .
$$

The latter condition means that $\mathbf{P}$ has full rank. One obtains

$$
\mathbf{P}_{r r}^{(9 \times 3)}=\left[\begin{array}{c}
-\tilde{\boldsymbol{d}}_{1} \\
-\tilde{\boldsymbol{d}}_{2} \\
-\tilde{\boldsymbol{d}}_{3}
\end{array}\right],
$$

where the following notation has been introduced:

$$
\tilde{\boldsymbol{w}}:=\left[\begin{array}{ccc}
0 & -w_{3} & w_{2} \\
w_{3} & 0 & -w_{1} \\
-w_{2} & w_{1} & 0
\end{array}\right] .
$$

That is, $\tilde{\boldsymbol{w}} \boldsymbol{v}=\boldsymbol{w} \times \boldsymbol{v}$ for any two spatial vectors $\boldsymbol{v}=\left[\begin{array}{lll}v_{1} & v_{2} & v_{3}\end{array}\right]^{T}$ and $\boldsymbol{w}=\left[\begin{array}{lll}w_{1} & w_{2} & w_{3}\end{array}\right]^{T}$. Note that (76) establishes a one-to-one correspondence of the vector space of spatial vectors $w \in \mathbb{R}^{3}$ and the matrix algebra of antisymmetric $3 \times 3$ matrices $\mathbf{W} \in \mathbb{R}^{3 \times 3}$. The inverse operation will be denoted by ax $(\cdot)$. That is,

$$
\mathbf{W}=\tilde{\boldsymbol{w}} \quad \Leftrightarrow \quad \boldsymbol{w}=\operatorname{ax}(\mathbf{W})
$$

Equivalently,

$$
W_{i j}=-\epsilon_{i j k} w_{k}, \quad w_{i}=-\epsilon_{i j k} W_{j k},
$$

where use has been made of the totally antisymmetric permutation symbol $\epsilon_{i j k}$ [22]. Further,

$$
\mathbf{P}_{f r}^{(9 M \times 3)}=\left[\begin{array}{c}
\mathbf{P}_{f r, 1} \\
\mathbf{P}_{f r, 2} \\
\vdots \\
\mathbf{P}_{f r, M}
\end{array}\right], \quad \mathbf{P}_{f f}^{(9 M \times M)}=\left[\begin{array}{cccc}
\boldsymbol{p}_{f f} & \mathbf{0} & \cdots & \mathbf{0} \\
\mathbf{0} & \boldsymbol{p}_{f f} & \cdots & \mathbf{0} \\
\vdots & \vdots & \ddots & \mathbf{0} \\
\mathbf{0} & \mathbf{0} & \cdots & \boldsymbol{p}_{f f}
\end{array}\right]
$$

with

$$
\mathbf{P}_{f r, \mu}^{(9 \times 3)}=\left[\begin{array}{c}
-\tilde{\boldsymbol{q}}_{\mu 1} \\
-\tilde{\boldsymbol{q}}_{\mu 2} \\
-\tilde{\boldsymbol{q}}_{\mu 3}
\end{array}\right], \quad \boldsymbol{p}_{f f}^{(9 \times 1)}=\left[\begin{array}{l}
\boldsymbol{d}_{1} \\
\boldsymbol{d}_{2} \\
\boldsymbol{d}_{3}
\end{array}\right] .
$$

Considering (46) and (75), $\mathbf{P}_{f r, \mu}=q_{f, \mu}^{\prime} \mathbf{P}_{r r}$. Making use of the Kronecker matrix product,

$$
\mathbf{P}_{f r}=\boldsymbol{q}_{f}^{\prime} \otimes \mathbf{P}_{r r}, \quad \mathbf{P}_{f f}=\mathbf{I}_{M} \otimes \boldsymbol{p}_{f f} .
$$




\section{Null-space projection of the GCMS formulation}

In [8], it has been shown that the ACF and the FFRF are equivalent on the level of the continuum theory, since both formulations are derived from D'Alemberts principle applying the same assumptions (small deformations with respect to a co-rotational reference configuration, e.g.). In addition, it has been demonstrated on the basis of a two-dimensional example that the two formulations lead to exactly the same numerical results if the same co-rotational reference configurations are used.

Particularly regarding computer implementations, it is an important characteristic of the ACF that no shape integrals appear in the formulation other than the ones constituting the standard finite element mass and the stiffness matrix, which is in contrast to the FFRF where additional inertia integrals appear. Therefore, two questions arise:

1. How can the equivalence mentioned before also be established at the discrete level?

2. How can this equivalence be exploited to relate the FFRF inertia integrals with the finite element mass matrix?

Answers to both questions are given in this section. It is explicated in detail how the null-space projection method can be employed to derive the modally reduced equations of motion of the FFRF from the ones of the GCMS, i.e. the modally reduced ACF. A comparison of the so-obtained formulas with the standard expressions of FFRF reveals the aspired relations between the FFRF inertia integrals and the finite element mass matrix. This leads to a potentially simplified implementation of FFRF, eliminating the necessity of performing extra calculations (shape integral evaluations) at the level of the element routine and consequently to a possible decoupling of the FFRF-related implementation from the finite element code.

\subsection{Constrained equations of motion}

The projection method can be applied to eliminate the ambiguity which is inherent to the original GCMS formulation due to the fact that no constraint conditions are imposed on the nine rotational and $9 M$ flexible degrees of freedom, which is in contrast to the FFRF. For the derivation of the projected equations of motion, without loss of generality, it can be assumed that the rigid body constraints (65) are fulfilled a priori. As a consequence, $\boldsymbol{q}_{r}=\boldsymbol{q}_{r}^{*}$ in (60) and $\partial A_{i j} / \partial \boldsymbol{q}_{f}=\mathbf{0}$ in (61). Thus,

$$
\boldsymbol{q}_{\mathrm{flex}}=\left[\begin{array}{c}
\mathbf{0} \\
\mathbf{0} \\
\boldsymbol{q}_{f}
\end{array}\right], \quad \hat{\boldsymbol{f}}_{\mathrm{nl}}=\left[\begin{array}{c}
\mathbf{0} \\
\boldsymbol{f}_{\mathrm{nl}} \\
\mathbf{0}
\end{array}\right] .
$$

It is beneficial to introduce the notation

$$
\overline{\mathbf{K}}_{t t}=\boldsymbol{\Phi}_{t}^{T} \mathbf{K} \boldsymbol{\Phi}_{t}, \quad \overline{\mathbf{K}}_{t r}=\boldsymbol{\Phi}_{t}^{T} \mathbf{K} \boldsymbol{\Phi}_{r}, \ldots \quad \overline{\mathbf{K}}_{f f}=\boldsymbol{\Phi}_{f}^{T} \mathbf{K} \boldsymbol{\Phi}_{f} .
$$

and, analogously,

$$
\mathbf{M}_{t t}=\boldsymbol{\Phi}_{t}^{T} \mathbf{M} \boldsymbol{\Phi}_{t}, \quad \mathbf{M}_{t r}=\boldsymbol{\Phi}_{t}^{T} \mathbf{M} \boldsymbol{\Phi}_{r}, \ldots \quad \mathbf{M}_{f f}=\boldsymbol{\Phi}_{f}^{T} \mathbf{M} \boldsymbol{\Phi}_{f} .
$$

A purely translational motion, which is represented by $\boldsymbol{q}_{t}$, cannot give rise to internal forces. Therefore,

$$
\overline{\mathbf{K}}_{t t}=\mathbf{O}^{(3 \times 3)}, \quad \overline{\mathbf{K}}_{t r}=\mathbf{O}^{(3 \times 9)}, \quad \overline{\mathbf{K}}_{t f}=\mathbf{O}^{(3 \times 9 M)} .
$$

Equation (85) is also a consequence of the translational invariance of the FE stiffness matrix (see also the Appendix). Note that there is no analogous argument for the rotational stiffness components, since the nine rotational coordinates, $\boldsymbol{q}_{r}$, also subsume deformational degrees of freedom, a priori. Denoting the rotated stiffness sub-matrices by

$$
\hat{\mathbf{K}}_{r r}=\mathbf{A}_{\mathrm{bd}} \overline{\mathbf{K}}_{r r} \mathbf{A}_{\mathrm{bd}}^{T}, \quad \hat{\mathbf{K}}_{r f}=\mathbf{A}_{\mathrm{bd}} \overline{\mathbf{K}}_{r f} \mathbf{A}_{\mathrm{bd}}^{T}, \quad \hat{\mathbf{K}}_{f f}=\mathbf{A}_{\mathrm{bd}} \overline{\mathbf{K}}_{f f} \mathbf{A}_{\mathrm{bd}}^{T} .
$$

with properly adjusted block diagonal rotation matrices $\mathbf{A}_{\mathrm{bd}}$, the GCMS equations of motion (59) become

$$
\left[\begin{array}{lll}
\mathbf{M}_{t t} & \mathbf{M}_{t r} & \mathbf{M}_{t f} \\
\mathbf{M}_{t r}^{T} & \mathbf{M}_{r r} & \mathbf{M}_{r} f \\
\mathbf{M}_{t f}^{T} & \mathbf{M}_{r f}^{T} & \mathbf{M}_{f f}
\end{array}\right]\left[\begin{array}{c}
\ddot{\boldsymbol{q}}_{t} \\
\ddot{\boldsymbol{q}}_{r} \\
\ddot{\boldsymbol{q}}_{f}
\end{array}\right]+\left[\begin{array}{ccc}
\mathbf{O} & \mathbf{O} & \mathbf{O} \\
\mathbf{O} & \hat{\mathbf{K}}_{r r} & \hat{\mathbf{K}}_{r f} \\
\mathbf{O} & \hat{\mathbf{K}}_{r f}^{T} & \hat{\mathbf{K}}_{f f}
\end{array}\right]\left[\begin{array}{c}
\mathbf{0} \\
\mathbf{0} \\
\boldsymbol{q}_{f}
\end{array}\right]+\left[\begin{array}{c}
\mathbf{0} \\
\boldsymbol{f}_{\mathrm{nl}} \\
\mathbf{0}
\end{array}\right]+\mathbf{C}_{\lambda}^{T} \boldsymbol{\lambda}=\hat{\boldsymbol{f}}_{\mathrm{ext}} .
$$

Therein, the problem dependent constraint Jacobian reads

$$
\mathbf{C}_{\lambda}=\left[\begin{array}{lll}
\mathbf{C}_{b t} & \mathbf{C}_{b r} & \mathbf{C}_{b f}
\end{array}\right] .
$$




\subsection{Generalized velocities}

The mutually independent velocities $\boldsymbol{w}_{t} \in \mathbb{R}^{3}, \boldsymbol{w}_{r} \in \mathbb{R}^{3}$, and $\boldsymbol{w}_{f} \in \mathbb{R}^{M}$ are obtained from the generalized velocities $\dot{\boldsymbol{q}}=\left[\begin{array}{lll}\dot{\boldsymbol{q}}_{t} & \dot{\boldsymbol{q}}_{r} & \dot{\boldsymbol{q}}_{f}\end{array}\right]$ via

$$
\left[\begin{array}{c}
\dot{\boldsymbol{q}}_{t} \\
\dot{\boldsymbol{q}}_{r} \\
\dot{\boldsymbol{q}}_{f}
\end{array}\right]=\left[\begin{array}{ccc}
\mathbf{I} & \mathbf{O} & \mathbf{O} \\
\mathbf{O} & \mathbf{P}_{r r} & \mathbf{O} \\
\mathbf{O} & \mathbf{P}_{f r} & \mathbf{P}_{f f}
\end{array}\right]\left[\begin{array}{c}
\boldsymbol{w}_{t} \\
\boldsymbol{w}_{r} \\
\boldsymbol{w}_{f}
\end{array}\right] .
$$

Since the projection matrices have been chosen such that they have full rank, the velocity vector $\boldsymbol{w}=$ $\left[\begin{array}{lll}\boldsymbol{w}_{t} & \boldsymbol{w}_{r} & \boldsymbol{w}_{f}\end{array}\right]$ is uniquely determined by (89). Further, Eq. (89) implies that the rigid body constraint conditions

$$
\boldsymbol{C}(\boldsymbol{q})=\left[\begin{array}{l}
\boldsymbol{C}_{r}(\boldsymbol{q}) \\
\boldsymbol{C}_{f}(\boldsymbol{q})
\end{array}\right]=\mathbf{0}
$$

are fulfilled automatically at the velocity level,

$$
\dot{\boldsymbol{C}}(\boldsymbol{q})=\frac{\partial \boldsymbol{C}}{\partial \boldsymbol{q}} \dot{\boldsymbol{q}}=\mathbf{C} \dot{\boldsymbol{q}}=\mathbf{C P} \boldsymbol{w}=\mathbf{0}
$$

While $\boldsymbol{w}_{t}=\dot{\boldsymbol{q}}_{t}$, the interpretation of the rotational velocity $\boldsymbol{w}_{r}$ follows from $\dot{\boldsymbol{q}}_{r}=\mathbf{P}_{r r} \boldsymbol{w}_{r}$, the first three rows of which read

$$
\dot{\boldsymbol{d}}_{1}=-\tilde{\boldsymbol{d}}_{1} \boldsymbol{w}_{r}=\boldsymbol{w}_{r} \times \boldsymbol{d}_{1} .
$$

Analogous relations hold for rows 4,5,6 and 7,8,9, respectively. Together they constitute the definition of the angular velocity vector. That is,

$$
\boldsymbol{w}_{r}=\omega
$$

The interpretation of $\boldsymbol{w}_{f}$ is simple,

$$
\boldsymbol{w}_{f}=\dot{\boldsymbol{q}}_{f}^{\prime}=\left[\begin{array}{lll}
\dot{q}_{f, 1}^{\prime} & \cdots & \dot{q}_{f, M}^{\prime}
\end{array}\right]^{T},
$$

where the $q_{f, j}^{\prime}$ are the flexible coordinates of the FFRF.

The proof relies on the observation that

$$
\boldsymbol{q}_{f}=\mathbf{P}_{f f} \boldsymbol{q}_{f}^{\prime},
$$

which can be verified by direct calculation: For the case of two flexible modes,

$$
\mathbf{P}_{f f} \boldsymbol{q}_{f}^{\prime}=\left[\begin{array}{cc}
\boldsymbol{d}_{1} & \mathbf{0} \\
\boldsymbol{d}_{2} & \mathbf{0} \\
\boldsymbol{d}_{3} & \mathbf{0} \\
\mathbf{0} & \boldsymbol{d}_{1} \\
\mathbf{0} & \boldsymbol{d}_{2} \\
\mathbf{0} & \boldsymbol{d}_{3}
\end{array}\right]\left[\begin{array}{l}
q_{f, 1}^{\prime} \\
q_{f, 2}^{\prime}
\end{array}\right]=\left[\begin{array}{l}
q_{f, 1}^{\prime} \boldsymbol{d}_{1} \\
q_{f, 1}^{\prime} \boldsymbol{d}_{2} \\
q_{f, 1}^{\prime} \boldsymbol{d}_{3} \\
q_{f, 2}^{\prime} \boldsymbol{d}_{1} \\
q_{f, 2}^{\prime} \boldsymbol{d}_{2} \\
q_{f, 2}^{\prime} \boldsymbol{d}_{3}
\end{array}\right]=\left[\begin{array}{l}
\boldsymbol{q}_{11} \\
\boldsymbol{q}_{12} \\
\boldsymbol{q}_{13} \\
\boldsymbol{q}_{21} \\
\boldsymbol{q}_{22} \\
\boldsymbol{q}_{23}
\end{array}\right]=\boldsymbol{q}_{f} .
$$

The general result follows from a trivial generalization. In a similar manner, one can establish the helpful relation

$$
\dot{\mathbf{P}}_{f f} \boldsymbol{q}_{f}^{\prime}=\mathbf{P}_{f r} \boldsymbol{w}_{r}
$$

Namely,

$$
\dot{\mathbf{P}}_{f f} \boldsymbol{q}_{f}^{\prime}=\left[\begin{array}{cc}
\dot{\boldsymbol{d}}_{1} & \mathbf{0} \\
\dot{\boldsymbol{d}}_{2} & \mathbf{0} \\
\dot{\boldsymbol{d}}_{3} & \mathbf{0} \\
\mathbf{0} & \dot{\boldsymbol{d}}_{1} \\
\mathbf{0} & \dot{\boldsymbol{d}}_{2} \\
\mathbf{0} & \dot{\boldsymbol{d}}_{3}
\end{array}\right]\left[\begin{array}{c}
q_{f, 1}^{\prime} \\
q_{f, 2}^{\prime}
\end{array}\right]=\left[\begin{array}{c}
q_{f, 1}^{\prime} \dot{\boldsymbol{d}}_{1} \\
q_{f, 1}^{\prime} \dot{\boldsymbol{d}}_{2} \\
q_{f, 1}^{\prime} \dot{\boldsymbol{d}}_{3} \\
q_{f, 2}^{\prime} \dot{\boldsymbol{d}}_{1} \\
q_{f, 2}^{\prime} \dot{\boldsymbol{d}}_{2} \\
q_{f, 2}^{\prime} \dot{\boldsymbol{d}}_{3}
\end{array}\right]=-\left[\begin{array}{c}
q_{f, 1}^{\prime} \tilde{\boldsymbol{d}}_{1} \\
q_{f, 1}^{\prime} \tilde{\boldsymbol{d}}_{2} \\
q_{f, 1}^{\prime} \tilde{\boldsymbol{d}}_{3} \\
q_{f, 2}^{\prime} \tilde{\boldsymbol{d}}_{1} \\
q_{f, 2}^{\prime} \tilde{\boldsymbol{d}}_{2} \\
q_{f, 2}^{\prime} \tilde{\boldsymbol{d}}_{3}
\end{array}\right] \boldsymbol{w}_{r}=\mathbf{P}_{f r} \boldsymbol{w}_{r}
$$

Proof of (94): On the one hand,

$$
\dot{\boldsymbol{q}}_{f}=\mathbf{P}_{f r} \boldsymbol{w}_{r}+\mathbf{P}_{f f} \boldsymbol{w}_{f}
$$


according to (89). On the other hand, from (95),

$$
\dot{\boldsymbol{q}}_{f}=\dot{\mathbf{P}}_{f f} \boldsymbol{q}_{f}^{\prime}+\mathbf{P}_{f f} \dot{\boldsymbol{q}}_{f}^{\prime} .
$$

Making use of (97), $\mathbf{P}_{f f}\left(\boldsymbol{w}_{f}-\dot{\boldsymbol{q}}_{f}^{\prime}\right)=\mathbf{0}$. The result follows, since $\mathbf{P}_{f f}$ has full rank, i.e. $\frac{1}{3} \mathbf{P}_{f f}^{T}$ is a left inverse.

\subsection{Generalized accelerations}

The generalized accelerations are obtained from the time derivative of (89),

$$
\left[\begin{array}{c}
\ddot{\boldsymbol{q}}_{t} \\
\ddot{\boldsymbol{q}}_{r} \\
\ddot{\boldsymbol{q}}_{f}
\end{array}\right]=\left[\begin{array}{ccc}
\mathbf{I} & \mathbf{O} & \mathbf{O} \\
\mathbf{O} & \mathbf{P}_{r r} & \mathbf{O} \\
\mathbf{O} & \mathbf{P}_{f r} & \mathbf{P}_{f f}
\end{array}\right]\left[\begin{array}{c}
\dot{\boldsymbol{w}}_{t} \\
\dot{\boldsymbol{w}}_{r} \\
\dot{\boldsymbol{w}}_{f}
\end{array}\right]+\left[\begin{array}{ccc}
\mathbf{O} & \mathbf{O} & \mathbf{O} \\
\mathbf{O} & \dot{\mathbf{P}}_{r r} & \mathbf{O} \\
\mathbf{O} & \dot{\mathbf{P}}_{f r} & \dot{\mathbf{P}}_{f f}
\end{array}\right]\left[\begin{array}{c}
\boldsymbol{w}_{t} \\
\boldsymbol{w}_{r} \\
\boldsymbol{w}_{f}
\end{array}\right]
$$

Subsequently, suitable expressions for the time derivatives of the projection matrices are derived,

$$
\dot{\mathbf{P}}_{r r}=-\left[\begin{array}{c}
\dot{\tilde{d}}_{1} \\
\dot{\tilde{d}}_{2} \\
\dot{\tilde{d}}_{3}
\end{array}\right]=-\left[\begin{array}{c}
\widetilde{\omega \times d_{1}} \\
\widetilde{\omega \times d_{2}} \\
\widetilde{\omega \times d_{3}}
\end{array}\right]
$$

and, since $w_{r}=\omega$ and $-\left(\omega \times d_{i}\right) \times \omega=\omega \times\left(\omega \times d_{i}\right)=\tilde{\omega}^{2} d_{i}$,

$$
\dot{\mathbf{P}}_{r r} \boldsymbol{w}_{r}=\left[\begin{array}{l}
\tilde{\boldsymbol{\omega}}^{2} \boldsymbol{d}_{1} \\
\tilde{\boldsymbol{\omega}}^{2} \boldsymbol{d}_{2} \\
\tilde{\boldsymbol{\omega}}^{2} \boldsymbol{d}_{3}
\end{array}\right]=\left[\begin{array}{l}
\tilde{\boldsymbol{\omega}} \dot{\boldsymbol{d}}_{1} \\
\tilde{\boldsymbol{\omega}} \dot{\boldsymbol{d}}_{2} \\
\tilde{\boldsymbol{\omega}} \dot{\boldsymbol{d}}_{3}
\end{array}\right]
$$

Further,

Additionally,

$$
\dot{\boldsymbol{p}}_{f f}=\left[\begin{array}{c}
{[1.1] \dot{\boldsymbol{d}}_{1}} \\
\dot{\boldsymbol{d}}_{2} \\
\dot{\boldsymbol{d}}_{3}
\end{array}\right]=\left[\begin{array}{l}
\tilde{\boldsymbol{\omega}} \boldsymbol{d}_{1} \\
\tilde{\boldsymbol{\omega}} \boldsymbol{d}_{2} \\
\tilde{\boldsymbol{\omega}} \boldsymbol{d}_{3}
\end{array}\right]
$$

$$
\dot{\boldsymbol{p}}_{f f}=\dot{\boldsymbol{q}}_{r}=\mathbf{P}_{r r} \boldsymbol{w}_{r}
$$

And finally, $\dot{\mathbf{P}}_{f r, \mu}=\dot{q}_{f, \mu}^{\prime} \mathbf{P}_{r r}+q_{f, \mu}^{\prime} \dot{\mathbf{P}}_{r r}$ or

$$
\dot{\mathbf{P}}_{f r}=\dot{\boldsymbol{q}}_{f}^{\prime} \otimes \mathbf{P}_{r r}+\boldsymbol{q}_{f}^{\prime} \otimes \dot{\mathbf{P}}_{r r}
$$

\subsection{Mass matrices}

Subsequently, the inertia components related to the independent velocities $\boldsymbol{w}_{t}, \boldsymbol{w}_{r}$, and $\boldsymbol{w}_{f}$ are derived. The definition of the mass matrix, Eq. (4), together with

$$
\mathbf{N}^{\mathrm{FE}} \boldsymbol{\Phi}_{t}=\left[\begin{array}{lll}
N_{1}^{\mathrm{FE}} \mathbf{I} \cdots N_{N}^{\mathrm{FE}} \mathbf{I}
\end{array}\right]\left[\begin{array}{c}
\mathbf{I} \\
\vdots \\
\mathbf{I}
\end{array}\right]=\underbrace{\sum_{n=1}^{N} N_{n}^{\mathrm{FE}} \mathbf{I}}_{=1}
$$

yields

$$
\mathbf{M}_{t t}=\boldsymbol{\Phi}_{t}^{T} \mathbf{M} \boldsymbol{\Phi}_{t}=m \mathbf{I},
$$

with $m=\int_{V} \rho \mathrm{d} V$ being the total mass of the body.

Similarly, recognizing that

$$
x^{i}=\boldsymbol{e}_{i} \cdot \boldsymbol{x}=\sum_{n=1}^{N} N_{n}^{\mathrm{FE}}(\boldsymbol{x}) x_{n}^{i}
$$


and the definition of $\boldsymbol{\Phi}_{r}$, see (35), one obtains

$$
\mathbf{N}^{\mathrm{FE}} \boldsymbol{\Phi}_{r}=\left[N_{1}^{\mathrm{FE}} \mathbf{I} \cdots N_{N}^{\mathrm{FE}} \mathbf{I}\right]\left[\begin{array}{ccc}
x_{1}^{1} \mathbf{I} & x_{1}^{2} \mathbf{I} & x_{1}^{3} \mathbf{I} \\
\vdots & \vdots & \vdots \\
x_{N}^{1} \mathbf{I} & x_{N}^{2} \mathbf{I} & x_{N}^{3} \mathbf{I}
\end{array}\right]=\left[\begin{array}{lll}
x^{1} \mathbf{I} & x^{2} \mathbf{I} & x^{3} \mathbf{I}
\end{array}\right]
$$

and thus

$$
\mathbf{M}_{t r}=\boldsymbol{\Phi}_{t}^{T} \mathbf{M} \boldsymbol{\Phi}_{r}=m\left[\begin{array}{lll}
a^{1} \mathbf{I} & a^{2} \mathbf{I} & a^{3} \mathbf{I}
\end{array}\right],
$$

where

$$
a^{i}=\frac{1}{m} \int_{V} x^{i} \rho(\boldsymbol{x}) \mathrm{d} V
$$

denotes the $i$ th coordinate of the centre of mass of the body. Note that the volume integrals (112), as well as $(114,122,125,131)$, are just stated to enable a physical interpretation; they never need to be calculated explicitly. Regarding a computer implementation, they can be obtained from $(120,126,127,132)$ instead.

Analogous manipulations yield

$$
\mathbf{M}_{t f, \mu}=\boldsymbol{\Phi}_{t}^{T} \mathbf{M} \boldsymbol{\Phi}_{f, \mu}=m\left[\begin{array}{lll}
b_{\mu}^{1} \mathbf{I} & b_{\mu}^{2} \mathbf{I} & b_{\mu}^{3} \mathbf{I}
\end{array}\right] .
$$

Again, for a physical interpretation, it is possible to give integral expressions for the $b_{\mu}^{i}$, namely, being the weighted-mean amplitude of the $\mu$ th flexible mode,

$$
b_{\mu}^{i}=\frac{1}{m} \int_{V} \bar{\phi}_{f, \mu}^{i}(\boldsymbol{x}) \rho(\boldsymbol{x}) \mathrm{d} V .
$$

Therefore, the interpolated components of the flexible modes

$$
\bar{\phi}_{f, \mu}^{i}=\sum_{n=1}^{N} N_{n}^{\mathrm{FE}}(\boldsymbol{x}) \phi_{f, \mu}^{i}\left(\boldsymbol{x}_{n}\right)
$$

have been introduced according to the structure of $\boldsymbol{\Phi}_{f, \mu}$, see (52).

Finally,

$$
\mathbf{M}_{t f}=\boldsymbol{\Phi}_{t}^{T} \mathbf{M} \boldsymbol{\Phi}_{f}=\left[\begin{array}{lll}
\mathbf{M}_{t f, 1} & \ldots & \mathbf{M}_{t f, M}
\end{array}\right] .
$$

For the numerical calculation of the quantities $m, \overline{\boldsymbol{a}}$, and $\overline{\boldsymbol{b}}_{\mu}$, where

$$
\overline{\boldsymbol{a}}=\sum_{i=1}^{3} a^{i} \boldsymbol{e}_{i}, \quad \overline{\boldsymbol{b}}_{\mu}=\sum_{i=1}^{3} b_{\mu}^{i} \boldsymbol{e}_{i}
$$

it is convenient to introduce the reduced projection matrices ${ }^{1}$

$$
\boldsymbol{\phi}_{t}=\left[\begin{array}{c}
\boldsymbol{e}_{1} \\
\vdots \\
\boldsymbol{e}_{1}
\end{array}\right], \quad \boldsymbol{\phi}_{r}=\left[\begin{array}{ccc}
x_{1}^{1} \boldsymbol{e}_{1} & x_{1}^{2} \boldsymbol{e}_{1} & x_{1}^{3} \boldsymbol{e}_{1} \\
\vdots & \vdots & \vdots \\
x_{N}^{1} \boldsymbol{e}_{1} & x_{N}^{2} \boldsymbol{e}_{1} & x_{N}^{3} \boldsymbol{e}_{1}
\end{array}\right]
$$

of dimensions $3 N \times 1$ and $3 N \times 3$, respectively, and

$$
\boldsymbol{\phi}_{f, \mu}=\left[\begin{array}{ccc}
\phi_{f, \mu}^{1}\left(\boldsymbol{x}_{1}\right) \boldsymbol{e}_{1} & \phi_{f, \mu}^{2}\left(\boldsymbol{x}_{1}\right) \boldsymbol{e}_{1} & \phi_{f, \mu}^{3}\left(\boldsymbol{x}_{1}\right) \boldsymbol{e}_{1} \\
\vdots & \vdots & \vdots \\
\phi_{f, \mu}^{1}\left(\boldsymbol{x}_{N}\right) \boldsymbol{e}_{1} & \phi_{f, \mu}^{2}\left(\boldsymbol{x}_{N}\right) \boldsymbol{e}_{1} & \phi_{f, \mu}^{3}\left(\boldsymbol{x}_{N}\right) \boldsymbol{e}_{1}
\end{array}\right]
$$

of dimensions $3 N \times 3$. Consequently,

$$
m=\boldsymbol{\phi}_{t}^{T} \mathbf{M} \boldsymbol{\phi}_{t}, \quad m \overline{\boldsymbol{a}}^{T}=\boldsymbol{\phi}_{t}^{T} \mathbf{M} \boldsymbol{\phi}_{r}, \quad m \overline{\boldsymbol{b}}_{\mu}^{T}=\boldsymbol{\phi}_{t}^{T} \mathbf{M} \boldsymbol{\phi}_{f, \mu} .
$$

\footnotetext{
${ }^{1}$ Clearly, for the definition of $\boldsymbol{\phi}_{t}, \boldsymbol{\phi}_{r}$, and $\boldsymbol{\phi}_{f, \mu}$ the basis vectors $\boldsymbol{e}_{2}$ or $\boldsymbol{e}_{3}$ could have been used instead of $\boldsymbol{e}_{1}$ as well.
} 
Similarly, (4) and (110) yield

$$
\mathbf{M}_{r r}=\boldsymbol{\Phi}_{r}^{T} \mathbf{M} \boldsymbol{\Phi}_{r}=\left[\begin{array}{lll}
\vartheta^{11} \mathbf{I} & \vartheta^{12} \mathbf{I} & \vartheta^{13} \mathbf{I} \\
\vartheta^{21} \mathbf{I} & \vartheta^{22} \mathbf{I} & \vartheta^{23} \mathbf{I} \\
\vartheta^{31} \mathbf{I} & \vartheta^{32} \mathbf{I} & \vartheta^{33} \mathbf{I}
\end{array}\right] .
$$

According to the definition of $\boldsymbol{\Phi}_{r}$, see (35),

$$
\vartheta^{i j}=\int_{V} x_{i} x_{j} \rho(\boldsymbol{x}) \mathrm{d} V=\vartheta^{j i}
$$

Analogously,

$$
\mathbf{M}_{r} f=\boldsymbol{\Phi}_{r}^{T} \mathbf{M} \boldsymbol{\Phi}_{f}=\left[\mathbf{M}_{1} \ldots \mathbf{M}_{M}\right]
$$

is identified to consist of sub-matrices

$$
\mathbf{M}_{\mu}=\boldsymbol{\Phi}_{r}^{T} \mathbf{M} \boldsymbol{\Phi}_{f, \mu}=\left[\begin{array}{lll}
\vartheta_{\mu}^{11} \mathbf{I} & \vartheta_{\mu}^{12} \mathbf{I} & \vartheta_{\mu}^{13} \mathbf{I} \\
\vartheta_{\mu}^{21} \mathbf{I} & \vartheta_{\mu}^{22} \mathbf{I} & \vartheta_{\mu}^{23} \mathbf{I} \\
\vartheta_{\mu}^{31} \mathbf{I} & \vartheta_{\mu}^{32} \mathbf{I} & \vartheta_{\mu}^{33} \mathbf{I}
\end{array}\right]
$$

Again, according to the definitions of $\boldsymbol{\Phi}_{r}$ and $\boldsymbol{\Phi}_{f, \mu}$, Eqs. (35) and (52),

$$
\vartheta_{\mu}^{i j}=\int_{V} x^{i} \bar{\phi}_{f, \mu}^{j}(\boldsymbol{x}) \rho(\boldsymbol{x}) \mathrm{d} V \neq \vartheta_{\mu}^{j i}
$$

Regarding computer implementations, the inertia components $\vartheta^{i j}$ and $\vartheta_{\mu}^{i j}$ are obtained readily from the FE mass matrix via ${ }^{2}$

$$
\overline{\mathbf{T}}=\vartheta^{i j} \boldsymbol{e}_{i} \otimes \boldsymbol{e}_{j}=\boldsymbol{\phi}_{r}^{T} \mathbf{M} \boldsymbol{\phi}_{r}
$$

and

$$
\overline{\mathbf{T}}_{\mu}=\vartheta_{\mu}^{i j} \boldsymbol{e}_{i} \otimes \boldsymbol{e}_{j}=\boldsymbol{\phi}_{r}^{T} \mathbf{M} \boldsymbol{\phi}_{f, \mu} .
$$

From (126) on, the summation convention, commonly applied in the context of tensor calculus [22], will be used. That is, whenever a spatial index $(i, j=1,2,3)$ appears twice in one algebraic term, once in an upper and once in a lower position, it is implied that a summation takes place. For example,

$$
a^{i} \boldsymbol{e}_{i}=\sum_{i=1}^{3} a^{i} \boldsymbol{e}_{i}, \quad \vartheta^{i j} \boldsymbol{e}_{i} \otimes \boldsymbol{e}_{j}=\sum_{i, j=1}^{3} \vartheta^{i j} \boldsymbol{e}_{i} \otimes \boldsymbol{e}_{j} .
$$

The flexible part of the CGMS mass sub-matrix becomes

$$
\mathbf{M}_{f f}=\boldsymbol{\Phi}_{f}^{T} \mathbf{M} \boldsymbol{\Phi}_{f}=\left[\begin{array}{ccc}
\mathbf{M}_{11} & \ldots & \mathbf{M}_{1 M} \\
\vdots & \ddots & \vdots \\
\mathbf{M}_{M 1}^{T} & \ldots & \mathbf{M}_{M M}
\end{array}\right]
$$

with

$$
\mathbf{M}_{\mu \nu}=\left[\begin{array}{lll}
\vartheta_{\mu \nu}^{11} \mathbf{I} & \vartheta_{\mu \nu}^{12} \mathbf{I} & \vartheta_{\mu \nu}^{13} \mathbf{I} \\
\vartheta_{\mu \nu}^{21} \mathbf{I} & \vartheta_{\mu \nu}^{22} \mathbf{I} & \vartheta_{\mu \nu}^{23} \mathbf{I} \\
\vartheta_{\mu \nu}^{31} \mathbf{I} & \vartheta_{\mu \nu}^{32} \mathbf{I} & \vartheta_{\mu \nu}^{33} \mathbf{I}
\end{array}\right]
$$

and

$$
\vartheta_{\mu \nu}^{i j}=\int_{V} \bar{\phi}_{f, \mu}^{i}(\boldsymbol{x}) \bar{\phi}_{f, \nu}^{j}(\boldsymbol{x}) \rho(\boldsymbol{x}) \mathrm{d} V=\vartheta_{\nu \mu}^{j i}
$$

The corresponding Euler tensors are again readily obtained from the finite element mass matrix

$$
\overline{\mathbf{T}}_{\mu \nu}=\vartheta_{\mu \nu}^{i j} \boldsymbol{e}_{i} \otimes \boldsymbol{e}_{j}=\boldsymbol{\phi}_{f, \mu}^{T} \mathbf{M} \boldsymbol{\phi}_{f, \nu} .
$$

\footnotetext{
2 The tensor $\overline{\mathbf{T}}$ is sometimes called the (co-rotational) Euler tensor.
} 
7.5 Generalized inertia tensors

It will turn out that in the course of the null-space projection, the GCMS mass sub-matrices transform according to

$$
\begin{aligned}
& \mathbf{P}_{r r}^{T} \mathbf{M}_{r r} \mathbf{P}_{r r}=\left[\begin{array}{lll}
\tilde{\boldsymbol{d}}_{1} & \tilde{\boldsymbol{d}}_{2} \tilde{\boldsymbol{d}}_{3}
\end{array}\right]\left[\begin{array}{ccc}
\vartheta^{11} \mathbf{I} & \vartheta^{12} \mathbf{I} & \cdot \\
\vartheta^{21} \mathbf{I} & \cdot & \cdot \\
\cdot & \cdot & \cdot
\end{array}\right]\left[\begin{array}{l}
\tilde{\boldsymbol{d}}_{1} \\
\tilde{\boldsymbol{d}}_{2} \\
\tilde{\boldsymbol{d}}_{3}
\end{array}\right] \\
& =\sum_{i, j=1}^{3} \vartheta^{i j} \tilde{\boldsymbol{d}}_{i} \tilde{\boldsymbol{d}}_{j}=\vartheta^{i j}\left[\left(\boldsymbol{d}_{j} \cdot \boldsymbol{d}_{i}\right) \mathbf{I}-\boldsymbol{d}_{j} \otimes \boldsymbol{d}_{i}\right] \\
& =\vartheta^{i i} \mathbf{I}-\vartheta^{i j} \boldsymbol{d}_{i} \otimes \boldsymbol{d}_{j}=\boldsymbol{\Theta}^{(3 \times 3)}=\mathbf{A} \overline{\boldsymbol{\Theta}}_{r r} \mathbf{A}^{T} .
\end{aligned}
$$

That is, the projection of the rotational mass sub-matrix $\mathbf{M}_{r r}$ coincides with the common, spatial inertia tensor $\boldsymbol{\Theta}$. Since $\boldsymbol{d}_{j} \cdot \boldsymbol{d}_{i}=\delta_{j i}$, i.e. the Kronecker symbol, ${ }^{3}$

$$
\vartheta^{i j} \boldsymbol{d}_{j} \cdot \boldsymbol{d}_{i}=\vartheta^{i i}=\sum_{i=1}^{3} \vartheta^{i i}=\operatorname{trace} \mathbf{T}
$$

is the trace of the spatial Euler tensor $\mathbf{T}=\mathbf{A} \overline{\mathbf{T}} \mathbf{A}^{T}$, introduced in (126). Further,

$$
\overline{\boldsymbol{\Theta}}=\vartheta^{i i} \mathbf{I}-\vartheta^{i j} \boldsymbol{e}_{i} \otimes \boldsymbol{e}_{j}=\mathbf{A}^{T} \boldsymbol{\Theta}_{r r} \mathbf{A}
$$

is the co-rotational inertia tensor, which is constant in time.

Analogously,

$$
\mathbf{P}_{r r}^{T} \mathbf{M}_{\mu} \mathbf{P}_{r r}=\vartheta_{\mu}^{i i} \mathbf{I}-\vartheta_{\mu}^{i j} \boldsymbol{d}_{i} \otimes \boldsymbol{d}_{j}=\boldsymbol{\Theta}_{\mu}^{(3 \times 3)}=\mathbf{A} \overline{\boldsymbol{\Theta}}_{\mu} \mathbf{A}^{T}
$$

leads to the definition of a coupling inertia tensor related to the $\mu$ th flexible mode and of its co-rotational counterpart

$$
\overline{\boldsymbol{\Theta}}_{\mu}=\vartheta_{\mu}^{i i} \mathbf{I}-\vartheta_{\mu}^{i j} \boldsymbol{e}_{i} \otimes \boldsymbol{e}_{j}=\mathbf{A}^{T} \boldsymbol{\Theta}_{\mu} \mathbf{A}
$$

The inertia tensors related to the deformational motion are

$$
\mathbf{P}_{r r}^{T} \mathbf{M}_{\mu \nu} \mathbf{P}_{r r}=\vartheta_{\mu \nu}^{i i} \mathbf{I}-\vartheta_{\mu \nu}^{i j} \boldsymbol{d}_{i} \otimes \boldsymbol{d}_{j}=\boldsymbol{\Theta}_{\mu \nu}^{(3 \times 3)}=\mathbf{A} \overline{\boldsymbol{\Theta}}_{\mu \nu} \mathbf{A}^{T}
$$

and their co-rotational counterparts

$$
\overline{\boldsymbol{\Theta}}_{\mu \nu}=\vartheta_{\mu \nu}^{i i} \mathbf{I}-\vartheta_{\mu \nu}^{i j} \boldsymbol{e}_{i} \otimes \boldsymbol{e}_{j}=\mathbf{A}^{T} \boldsymbol{\Theta}_{\mu \nu} \mathbf{A}
$$

\subsection{Generalized 'inertia vectors'}

Additional inertia components related to the coupling of rotational and deformational motion arise from the transformation

$$
\begin{aligned}
\boldsymbol{\Xi}^{(3 \times M)} & :=\mathbf{P}_{r r}^{T} \mathbf{M}_{r} f \mathbf{P}_{f f}=\left[\mathbf{P}_{r r}^{T} \mathbf{M}_{1} \boldsymbol{p}_{f f} \ldots \mathbf{P}_{r r}^{T} \mathbf{M}_{M} \boldsymbol{p}_{f f}\right] \\
& =\left[\boldsymbol{\vartheta}_{1} \ldots \boldsymbol{\vartheta}_{M}\right]=\mathbf{A} \boldsymbol{\Xi}
\end{aligned}
$$

Therein,

$$
\mathbf{P}_{r r}^{T} \mathbf{M}_{\mu} \boldsymbol{p}_{f f}=\vartheta_{\mu}^{i j} \tilde{\boldsymbol{d}}_{i} \boldsymbol{d}_{j}=\vartheta_{\mu}^{i j} \boldsymbol{d}_{i} \times \boldsymbol{d}_{j}=\epsilon_{i j k} \vartheta_{\mu}^{i j} \boldsymbol{d}_{k}=\vartheta_{\mu}^{k} \boldsymbol{d}_{k}=\vartheta_{\mu},
$$

where use has been made of $\boldsymbol{d}_{i} \times \boldsymbol{d}_{j}=\epsilon_{i j k} \boldsymbol{d}_{k}$ and of the definition $\vartheta_{\mu}^{k}:=\epsilon_{k i j} \vartheta_{\mu}^{i j}$. The co-rotational counterparts are $\bar{\vartheta}_{\mu}:=\vartheta_{\mu}^{k} \boldsymbol{e}_{k}$ and

$$
\bar{\Xi}=\left[\bar{\vartheta}_{1} \ldots \bar{\vartheta}_{M}\right]=\mathbf{A}^{T} \Xi
$$

$$
{ }^{3} \delta_{i j}=\left\{\begin{array}{l}
1 \text { for } i=j \\
0 \text { for } i \neq j
\end{array}\right.
$$


The coupling inertia vectors $\boldsymbol{\vartheta}_{\mu}$ and tensors $\boldsymbol{\Theta}_{\mu}$ are related via

$$
\tilde{\vartheta}_{\mu}=\boldsymbol{\Theta}_{\mu}-\boldsymbol{\Theta}_{\mu}^{T} .
$$

Equivalently, making use of (77), $\boldsymbol{\vartheta}_{\mu}=\mathrm{ax}\left(\boldsymbol{\Theta}_{\mu}-\boldsymbol{\Theta}_{\mu}^{T}\right)$.

Proof: For any spatial vector $v$,

$$
\begin{aligned}
\tilde{\boldsymbol{\vartheta}}_{\mu} \boldsymbol{v} & =\vartheta_{\mu} \times \boldsymbol{v}=\vartheta_{\mu}^{i j}\left(\boldsymbol{d}_{i} \times \boldsymbol{d}_{j}\right) \times \boldsymbol{v} \\
& =\vartheta_{\mu}^{i j}\left[\boldsymbol{d}_{i} \times\left(\boldsymbol{d}_{j} \times \boldsymbol{v}\right)-\boldsymbol{d}_{j} \times\left(\boldsymbol{d}_{i} \times \boldsymbol{v}\right)\right]=\left(\vartheta_{\mu}^{i j}-\vartheta_{\mu}^{j i}\right) \boldsymbol{d}_{i} \times\left(\boldsymbol{d}_{j} \times \boldsymbol{v}\right) \\
& =\left(\vartheta_{\mu}^{i j}-\vartheta_{\mu}^{j i}\right) \tilde{\boldsymbol{d}}_{i} \tilde{\boldsymbol{d}}_{j} \boldsymbol{v}=\left(\vartheta_{\mu}^{i j}-\vartheta_{\mu}^{j i}\right) \boldsymbol{d}_{i} \otimes \boldsymbol{d}_{j} \cdot \boldsymbol{v},
\end{aligned}
$$

where use has been made of the Jacobi identity

$$
\boldsymbol{d}_{i} \times\left(\boldsymbol{d}_{j} \times \boldsymbol{v}\right)+\boldsymbol{d}_{j} \times\left(\boldsymbol{v} \times \boldsymbol{d}_{j}\right)+\boldsymbol{v} \times\left(\boldsymbol{d}_{j} \times \boldsymbol{d}_{i}\right)=\mathbf{0} .
$$

and of

$$
\tilde{\boldsymbol{d}}_{i} \tilde{\boldsymbol{d}}_{j}=\left(\boldsymbol{d}_{j} \cdot \boldsymbol{d}_{i}\right) \mathbf{I}-\boldsymbol{d}_{j} \otimes \boldsymbol{d}_{i} .
$$

Additional inertia vectors emerge from

$$
\mathbf{P}_{r r}^{T} \mathbf{M}_{\mu \nu} \boldsymbol{p}_{f f}=-\vartheta_{\mu \nu}^{i j} \tilde{\boldsymbol{d}}_{i}^{T} \dot{\boldsymbol{d}}_{j}=\vartheta_{\mu \nu}^{i j} \boldsymbol{d}_{i} \times \boldsymbol{d}_{j}=\vartheta_{\mu \nu}^{k} \boldsymbol{d}_{k}=: \vartheta_{\mu \nu}
$$

with components $\vartheta_{\mu \nu}^{k}=\epsilon_{i j k} \vartheta_{\mu \nu}^{i j}$. For $\mu=\nu, \vartheta_{m m}^{i j}$ is symmetric with respect to $i$ and $j$ and thus $\vartheta_{m m}^{k}=0$. Further,

$$
\boldsymbol{\vartheta}_{\mu \nu}=\vartheta_{\mu \nu}^{i j} \boldsymbol{d}_{i} \times \boldsymbol{d}_{j}=-\vartheta_{\nu \mu}^{j i} \boldsymbol{d}_{j} \times \boldsymbol{d}_{i}=-\boldsymbol{\vartheta}_{\nu \mu} .
$$

Their co-rotational counterparts are $\bar{\vartheta}_{\mu \nu}:=\vartheta_{\mu \nu}^{k} \boldsymbol{e}_{k}=\vartheta_{\mu \nu}^{i j} \boldsymbol{e}_{i} \times \boldsymbol{e}_{j}$. In analogy with (143), the inertia vectors $\vartheta_{\mu \nu}$ and tensors $\boldsymbol{\Theta}_{\mu \nu}$ are related via

$$
\tilde{\boldsymbol{\vartheta}}_{\mu \nu}=\boldsymbol{\Theta}_{\mu \nu}-\boldsymbol{\Theta}_{\mu \nu}^{T}
$$

or $\vartheta_{\mu \nu}=\operatorname{ax}\left(\boldsymbol{\Theta}_{\mu \nu}-\boldsymbol{\Theta}_{\mu \nu}^{T}\right)$. It will turn out that it is convenient to arrange the vectors $\vartheta_{\mu \nu}$ to obtain $3 \times M$ matrices

$$
\mathbf{\Xi}_{\mu}:=\left[\boldsymbol{\vartheta}_{\mu 1} \cdots \vartheta_{\mu M}\right], \quad \mu=1, \ldots, M .
$$

\subsection{Effects of eccentricity}

Concerning the translational components of the GCMS mass matrix, for any spatial vector,

$$
\mathbf{M}_{t r} \mathbf{P}_{r r} \boldsymbol{v}=m a^{i} \tilde{\boldsymbol{d}}_{i} \boldsymbol{v}=-m \boldsymbol{v} \times\left(a^{i} \boldsymbol{d}_{i}\right)=m \tilde{\boldsymbol{a}} \boldsymbol{v}
$$

and thus,

$$
\mathbf{M}_{t r} \mathbf{P}_{r r}=m \tilde{\boldsymbol{a}}=m \mathbf{A} \tilde{\overline{\boldsymbol{a}}} \mathbf{A}^{T}
$$

with $\boldsymbol{a}=a^{i} \boldsymbol{d}_{i}=\mathbf{A} \overline{\boldsymbol{a}}$ and $\overline{\boldsymbol{a}}=a^{i} \boldsymbol{e}_{i}$ being the spatial and the co-rotational position vector of the centre of mass.

Similarly, with $\boldsymbol{b}_{\mu}=b_{\mu}^{i} \boldsymbol{d}_{i}=\mathbf{A} \overline{\boldsymbol{b}}_{\mu}$ and $\overline{\boldsymbol{b}}_{\mu}=b_{\mu}^{i} \boldsymbol{e}_{i}$

$$
\mathbf{M}_{t f, \mu} \boldsymbol{p}_{f f}=m b_{\mu}^{i} \boldsymbol{d}_{i}=m \boldsymbol{b}_{\mu},
$$

and further, with $\mathbf{B}=\left[\begin{array}{lll}\boldsymbol{b}_{1} & \ldots & \boldsymbol{b}_{M}\end{array}\right]$ and $\overline{\mathbf{B}}=\left[\begin{array}{lll}\overline{\boldsymbol{b}}_{1} & \ldots & \overline{\boldsymbol{b}}_{M}\end{array}\right]$

$$
\mathbf{M}_{t f} \mathbf{P}_{f f}=m \mathbf{B}=m \mathbf{A} \overline{\mathbf{B}} .
$$

Finally,

$$
\mathbf{M}_{t f, \mu} \mathbf{P}_{r r}=-m b_{\mu}^{i} \tilde{\boldsymbol{d}}_{i}=-m \tilde{\boldsymbol{b}}_{\mu}=-m \mathbf{A} \tilde{\overline{\boldsymbol{b}}}_{\mu} \mathbf{A}^{T}
$$


7.8 Generalized inertia forces

Projecting the generalized inertia forces yields the expression

$$
\begin{aligned}
& {\left[\begin{array}{ccc}
\mathbf{I} & \mathbf{O} & \mathbf{O} \\
\mathbf{O} & \mathbf{P}_{r r}^{T} & \mathbf{P}_{f r}^{T} \\
\mathbf{O} & \mathbf{O} & \mathbf{P}_{f f}^{T}
\end{array}\right]\left[\begin{array}{ccc}
\mathbf{M}_{t t} & \mathbf{M}_{t r} & \mathbf{M}_{t f} \\
\cdot & \mathbf{M}_{r r} & \mathbf{M}_{r f} \\
\operatorname{sym} & \cdot & \mathbf{M}_{f f}
\end{array}\right]\left[\begin{array}{ccc}
\mathbf{I} & \mathbf{O} & \mathbf{O} \\
\mathbf{O} & \mathbf{P}_{r r} & \mathbf{O} \\
\mathbf{O} & \mathbf{P}_{f r} & \mathbf{P}_{f f}
\end{array}\right]\left[\begin{array}{c}
\dot{\boldsymbol{w}}_{t} \\
\dot{\boldsymbol{w}}_{r} \\
\dot{\boldsymbol{w}}_{f}
\end{array}\right]} \\
& =\left[\begin{array}{ccc}
\mathbf{M}_{t t} & \mathbf{M}_{t r}^{\prime} & \mathbf{M}_{t f}^{\prime} \\
\cdot & \mathbf{M}_{r r}^{\prime} & \mathbf{M}_{r} f^{\prime} \\
\operatorname{sym} & \cdot & \mathbf{M}_{f f}^{\prime}
\end{array}\right]\left[\begin{array}{c}
\dot{\boldsymbol{w}}_{t} \\
\dot{\boldsymbol{w}}_{r} \\
\dot{\boldsymbol{w}}_{f}
\end{array}\right]
\end{aligned}
$$

with the dimensionally reduced mass matrices

$$
\begin{aligned}
\mathbf{M}_{t r}^{\prime} & =\mathbf{M}_{t r} \mathbf{P}_{r r}+\mathbf{M}_{t f} \mathbf{P}_{f r} \\
\mathbf{M}_{t f}^{\prime} & =\mathbf{M}_{t f} \mathbf{P}_{f f} \\
\mathbf{M}_{r r}^{\prime} & =\mathbf{P}_{r r}^{T} \mathbf{M}_{r r} \mathbf{P}_{r r}+\mathbf{P}_{r r}^{T} \mathbf{M}_{r} f \mathbf{P}_{f r}+\mathbf{P}_{f r}^{T} \mathbf{M}_{r} f^{T} \mathbf{P}_{r r}+\mathbf{P}_{f r}^{T} \mathbf{M}_{f f} \mathbf{P}_{f r}, \\
\mathbf{M}_{r}^{\prime} & =\mathbf{P}_{r r}^{T} \mathbf{M}_{r} f \mathbf{P}_{f f}+\mathbf{P}_{f r}^{T} \mathbf{M}_{f f} \mathbf{P}_{f f} \\
\mathbf{M}_{f f}^{\prime} & =\mathbf{P}_{f f}^{T} \mathbf{M}_{f f} \mathbf{P}_{f f} .
\end{aligned}
$$

Each single term will be explicated subsequently:

$$
\begin{aligned}
& \mathbf{M}_{t r} \mathbf{P}_{r r}=m \tilde{\boldsymbol{a}}, \\
& \mathbf{M}_{t f} \mathbf{P}_{f r}=\sum_{\mu=1}^{M} q_{f, \mu}^{\prime} \mathbf{M}_{t f, \mu} \mathbf{P}_{r r}=-\sum_{\mu=1}^{M} q_{f, \mu}^{\prime} m \tilde{\boldsymbol{b}}_{\mu}=-m \widetilde{\mathbf{B} \boldsymbol{q}_{f}^{\prime}}, \\
& \mathbf{M}_{t f} \mathbf{P}_{f f}=m \mathbf{B}, \\
& \mathbf{P}_{r r}^{T} \mathbf{M}_{r r} \mathbf{P}_{r r}=\boldsymbol{\Theta}, \\
& \mathbf{P}_{r r}^{T} \mathbf{M}_{r} f \mathbf{P}_{f r}=\sum_{\mu=1}^{M} q_{f, \mu}^{\prime} \mathbf{P}_{r r}^{T} \mathbf{M}_{\mu} \mathbf{P}_{r r}=\sum_{\mu=1}^{M} q_{f, \mu}^{\prime} \mathbf{\Theta}_{\mu} \\
& \mathbf{P}_{f r}^{T} \mathbf{M}_{f f}^{T} \mathbf{P}_{f r}=\sum_{\mu, \nu=1}^{M} q_{f, \mu}^{\prime} q_{f, \nu}^{\prime} \mathbf{P}_{r r}^{T} \mathbf{M}_{\mu \nu} \mathbf{P}_{r r}=\sum_{\mu, \nu=1}^{M} q_{f, \mu}^{\prime} q_{f, \nu}^{\prime} \mathbf{\Theta}_{\mu \nu}, \\
& \mathbf{P}_{r r}^{T} \mathbf{M}_{r} f \mathbf{P}_{f f}=\mathbf{\Xi}, \\
& \mathbf{P}_{f r}^{T} \mathbf{M}_{f f}^{T} \mathbf{P}_{f f}=\sum_{\mu=1}^{M} q_{f, \mu}^{\prime} \mathbf{P}_{r r}^{T}\left[\mathbf{M}_{\mu 1} \boldsymbol{p}_{f f} \cdots \mathbf{M}_{\mu M} \boldsymbol{p}_{f f}\right]=\sum_{\mu=1}^{M} q_{f, \mu}^{\prime} \boldsymbol{\Xi}_{\mu}, \\
& \mathbf{P}_{f f}^{T} \mathbf{M}_{f f}^{T} \mathbf{P}_{f f}=\boldsymbol{\Theta}_{f f} .
\end{aligned}
$$

The last equation deserves additional attention. Defining

$$
\boldsymbol{\Theta}_{f f}:=\mathbf{P}_{f f}^{T} \mathbf{M}_{f f} \mathbf{P}_{f f}=\left[\begin{array}{ccc}
\mathbf{P}_{f f}^{T} \mathbf{M}_{11} \boldsymbol{p}_{f f} & \ldots & \mathbf{P}_{f f}^{T} \mathbf{M}_{1 M} \boldsymbol{p}_{f f} \\
\vdots & \ddots & \vdots \\
\mathbf{P}_{f f}^{T} \mathbf{M}_{M 1} \boldsymbol{p}_{f f} & \ldots & \mathbf{P}_{f f}^{T} \mathbf{M}_{M M} \boldsymbol{p}_{f f}
\end{array}\right]
$$

with

one obtains

$$
\mathbf{P}_{f f}^{T} \mathbf{M}_{\mu \nu} \boldsymbol{p}_{f f}=\vartheta_{\mu \nu}^{i j} \boldsymbol{d}_{i}^{T} \boldsymbol{d}_{j}=\vartheta_{\mu \nu}^{i i}=\operatorname{trace} \overline{\mathbf{T}}_{\mu \nu}
$$

$$
\boldsymbol{\Theta}_{f f}^{(M \times M)}=\left[\begin{array}{ccc}
\vartheta_{11}^{i i} & \cdots & \vartheta_{1 M}^{i i} \\
\vdots & \ddots & \vdots \\
\vartheta_{M 1}^{i i} & \cdots & \vartheta_{M M}^{i i}
\end{array}\right]=\overline{\boldsymbol{\Theta}}_{f f}
$$


According to (131), $\vartheta_{\mu \nu}^{i i}=\vartheta_{\nu \mu}^{i i}$, i.e. $\boldsymbol{\Theta}_{f f}$ is symmetric. Moreover, $\boldsymbol{\Theta}_{f f}$ is a diagonal matrix, if the flexible modes are chosen to be orthogonal, and coincides with the identity matrix, if they are orthonormal. Due to the invariance property of the trace operator, the spatial and the co-rotational appearances, $\boldsymbol{\Theta}_{f f}$ and $\overline{\boldsymbol{\Theta}}_{f f}$, coincide and are, thus, constant in time.

Concluding, the reduced mass sub-matrices read

$$
\begin{aligned}
\mathbf{M}_{t r}^{\prime} & =m\left(\tilde{\boldsymbol{a}}-q_{f, \mu}^{\prime} \tilde{\boldsymbol{b}}_{\mu}\right)=: m \tilde{\boldsymbol{r}}\left(\boldsymbol{q}_{f}^{\prime}\right), \\
\mathbf{M}_{t f}^{\prime} & =m \mathbf{B}, \\
\mathbf{M}_{r r}^{\prime} & =\boldsymbol{\Theta}+2 q_{f, \mu}^{\prime} \boldsymbol{\Theta}_{\mu}+q_{f, \mu}^{\prime} q_{f, \nu}^{\prime} \boldsymbol{\Theta}_{\mu \nu}=: \boldsymbol{\Theta}_{r r}\left(\boldsymbol{q}_{f}^{\prime}\right), \\
\mathbf{M}_{r} f^{\prime} & =\boldsymbol{\Xi}+q_{f, \mu}^{\prime} \boldsymbol{\Xi}_{\mu}=: \boldsymbol{\Theta}_{r} f\left(\boldsymbol{q}_{f}^{\prime}\right), \\
\mathbf{M}_{f f}^{\prime} & =\boldsymbol{\Theta}_{f f} .
\end{aligned}
$$

Therein, the summation convention has been applied also to the summation over the indices $\mu$ and $v$ denoting the mode number. That is, the summation symbols $\sum_{\mu}$ and $\sum_{v}$ are dropped in (163). It turns out that the total mass matrix evolves according to

$$
\left[\begin{array}{ccc}
m \mathbf{I} & m \tilde{\boldsymbol{r}} & m \mathbf{B} \\
\cdot & \boldsymbol{\Theta}_{r r} & \boldsymbol{\Theta}_{r} f \\
\operatorname{sym} & \cdot & \boldsymbol{\Theta}_{f f}
\end{array}\right]=\left[\begin{array}{ccc}
\mathbf{A} & \mathbf{O} & \mathbf{O} \\
\mathbf{O} & \mathbf{A} & \mathbf{O} \\
\mathbf{O} & \mathbf{O} & \mathbf{I}
\end{array}\right]\left[\begin{array}{ccc}
m \mathbf{I} & m \tilde{\bar{r}} & m \overline{\mathbf{B}} \\
\cdot & \overline{\boldsymbol{\Theta}}_{r r} & \overline{\boldsymbol{\Theta}}_{r f} \\
\operatorname{sym} & \cdot & \boldsymbol{\Theta}_{f f}
\end{array}\right]\left[\begin{array}{ccc}
\mathbf{A}^{T} & \mathbf{O} & \mathbf{O} \\
\mathbf{O} & \mathbf{A}^{T} & \mathbf{O} \\
\mathbf{O} & \mathbf{O} & \mathbf{I}
\end{array}\right] .
$$

Its co-rotational counterpart on the right-hand side solely depends on the reduced flexible coordinates $\boldsymbol{q}_{f}^{\prime}$ and is composed of the constant inertia components $m, m \overline{\boldsymbol{a}}, m \overline{\mathbf{B}}, \overline{\boldsymbol{\Theta}}$, etc., which can easily be determined from the FE mass matrix.

It is illustrative to introduce mass matrices related to the deformed configuration

$$
\begin{aligned}
\mathbf{M}_{t r}^{d}\left(\boldsymbol{q}_{f}^{\prime}\right) & :=\mathbf{M}_{t r}+q_{f, \nu}^{\prime} \mathbf{M}_{t f, v}, \\
\mathbf{M}_{r r}^{d}\left(\boldsymbol{q}_{f}^{\prime}\right) & :=\mathbf{M}_{r r}+2 q_{f, \mu}^{\prime} \mathbf{M}_{\mu}+q_{f, \mu}^{\prime} q_{f, \nu}^{\prime} \mathbf{M}_{\mu \nu}, \\
\mathbf{M}_{r f, \mu}^{d}\left(\boldsymbol{q}_{f}^{\prime}\right) & :=\mathbf{M}_{\mu}+q_{f, \nu}^{\prime} \mathbf{M}_{\mu \nu} .
\end{aligned}
$$

Doing so, the projection of the mass matrix obtains a plausible shape

$$
\left[\begin{array}{ccc}
m \mathbf{I} & m \tilde{\boldsymbol{r}} & m \mathbf{B} \\
\cdot & \boldsymbol{\Theta}_{r r} & \boldsymbol{\Theta}_{r f} \\
\operatorname{sym} & \cdot & \boldsymbol{\Theta}_{f f}
\end{array}\right]=\left[\begin{array}{ccc}
\mathbf{I} & \mathbf{O} & \mathbf{O} \\
\mathbf{O} & \mathbf{P}_{r r}^{T} & \mathbf{O} \\
\mathbf{O} & \mathbf{O} & \mathbf{P}_{f f}^{T}
\end{array}\right]\left[\begin{array}{ccc}
\mathbf{M}_{t t} & \mathbf{M}_{t r}^{d} & \mathbf{M}_{t f} \\
\cdot & \mathbf{M}_{r r}^{d} & \mathbf{M}_{r} f^{d} \\
\operatorname{sym} & \cdot & \mathbf{M}_{f f}
\end{array}\right]\left[\begin{array}{ccc}
\mathbf{I} & \mathbf{O} & \mathbf{O} \\
\mathbf{O} & \mathbf{P}_{r r} & \mathbf{O} \\
\mathbf{O} & \mathbf{O} & \mathbf{P}_{f f}
\end{array}\right]
$$

\subsection{Gyroscopic forces and moments}

Projecting the generalized gyroscopic forces originating from the second term on the right-hand side of (101) yields

$$
\left[\begin{array}{c}
\boldsymbol{Q}_{\omega t} \\
\boldsymbol{Q}_{\omega r} \\
\boldsymbol{Q}_{\omega f}
\end{array}\right]=\left[\begin{array}{ccc}
\mathbf{I} & \mathbf{O} & \mathbf{O} \\
\mathbf{O} & \mathbf{P}_{r r}^{T} & \mathbf{P}_{f r}^{T} \\
\mathbf{O} & \mathbf{O} & \mathbf{P}_{f f}^{T}
\end{array}\right]\left[\begin{array}{ccc}
\mathbf{M}_{t t} & \mathbf{M}_{t r} & \mathbf{M}_{t f} \\
\cdot & \mathbf{M}_{r r} & \mathbf{M}_{r f} \\
\operatorname{sym} & \cdot & \mathbf{M}_{f f}
\end{array}\right]\left[\begin{array}{ccc}
\mathbf{O} & \mathbf{O} & \mathbf{O} \\
\mathbf{O} & \dot{\mathbf{P}}_{r r} & \mathbf{O} \\
\mathbf{O} & \dot{\mathbf{P}}_{f r} & \dot{\mathbf{P}}_{f f}
\end{array}\right]\left[\begin{array}{c}
\boldsymbol{w}_{t} \\
\boldsymbol{w}_{r} \\
\boldsymbol{w}_{f}
\end{array}\right]
$$

That is,

$$
\begin{aligned}
\boldsymbol{Q}_{\omega t}= & \left(\mathbf{M}_{t r} \dot{\mathbf{P}}_{r r}+\mathbf{M}_{t f} \dot{\mathbf{P}}_{f r}\right) \boldsymbol{w}_{r}+\mathbf{M}_{t f} \dot{\mathbf{P}}_{f f} \boldsymbol{w}_{f} \\
= & \left(\mathbf{M}_{t r}+q_{f, v}^{\prime} \mathbf{M}_{t f, v}\right) \dot{\mathbf{P}}_{r r} \boldsymbol{w}_{r}+w_{f, v} \mathbf{M}_{t f, v}\left(\mathbf{P}_{r r} \boldsymbol{w}_{r}+\dot{\boldsymbol{p}}_{f f}\right), \\
\boldsymbol{Q}_{\omega r}= & \left(\mathbf{P}_{r r}^{T} \mathbf{M}_{r r} \dot{\mathbf{P}}_{r r}+\mathbf{P}_{r r}^{T} \mathbf{M}_{r} f \dot{\mathbf{P}}_{f r}+\mathbf{P}_{f r}^{T} \mathbf{M}_{r} f^{T} \dot{\mathbf{P}}_{r r}+\mathbf{P}_{f r}^{T} \mathbf{M}_{f f}^{T} \dot{\mathbf{P}}_{f r}\right) \boldsymbol{w}_{r} \\
& +\left(\mathbf{P}_{r r}^{T} \mathbf{M}_{r} f \dot{\mathbf{P}}_{f f}+\mathbf{P}_{f r}^{T} \mathbf{M}_{f f}^{T} \dot{\mathbf{P}}_{f f}\right) \boldsymbol{w}_{f} \\
= & \mathbf{P}_{r r}^{T}\left(\mathbf{M}_{r r}+2 q_{f, \mu}^{\prime} \mathbf{M}_{\mu}+q_{f, \mu}^{\prime} q_{f, v}^{\prime} \mathbf{M}_{\mu \nu}\right) \dot{\mathbf{P}}_{r r} \boldsymbol{w}_{r}
\end{aligned}
$$




$$
\begin{gathered}
+w_{f, \nu} \mathbf{P}_{r r}^{T}\left(\mathbf{M}_{v}+q_{f, \mu}^{\prime} \mathbf{M}_{\mu \nu}\right)\left(\mathbf{P}_{r r} \boldsymbol{w}_{r}+\dot{\boldsymbol{p}}_{f f}\right) \\
\boldsymbol{Q}_{\omega f}=\left(\mathbf{P}_{f f}^{T} \mathbf{M}_{r} f^{T} \dot{\mathbf{P}}_{r r}+\mathbf{P}_{f f}^{T} \mathbf{M}_{f f} \dot{\mathbf{P}}_{f r}\right) \boldsymbol{w}_{r}+\mathbf{P}_{f f}^{T} \mathbf{M}_{f f} \dot{\mathbf{P}}_{f f} \boldsymbol{w}_{f} .
\end{gathered}
$$

Further,

$$
\boldsymbol{Q}_{\omega f, \mu}=\boldsymbol{p}_{f f}^{T}\left(\mathbf{M}_{\mu}+q_{f, \nu}^{\prime} \mathbf{M}_{\mu \nu}\right) \dot{\mathbf{P}}_{r r} \boldsymbol{w}_{r}+w_{f, \nu} \boldsymbol{p}_{f f}^{T} \mathbf{M}_{\mu \nu}\left(\mathbf{P}_{r r} \boldsymbol{w}_{r}+\dot{\boldsymbol{p}}_{f f}\right) .
$$

With (165) and (105), more compact expressions are obtained:

$$
\begin{aligned}
& \boldsymbol{Q}_{\omega t}=\left(\mathbf{M}_{t r}^{d} \dot{\mathbf{P}}_{r r}+2\left(\mathbf{M}_{t f} \boldsymbol{w}_{f}\right) \mathbf{P}_{r r}\right) \boldsymbol{w}_{r}, \\
& \boldsymbol{Q}_{\omega r}=\left(\mathbf{P}_{r r}^{T} \mathbf{M}_{r r}^{d} \dot{\mathbf{P}}_{r r}+2 \mathbf{P}_{r r}^{T}\left(\mathbf{M}_{r} f^{d} \boldsymbol{w}_{f}\right) \mathbf{P}_{r r}\right) \boldsymbol{w}_{r}, \\
& \boldsymbol{Q}_{\omega f}=\left(\mathbf{P}_{f f}^{T}\left(\mathbf{M}_{r} f^{d}\right)^{T} \dot{\mathbf{P}}_{r r}+2 \mathbf{P}_{f f}^{T}\left(\mathbf{M}_{f f} \boldsymbol{w}_{f}\right) \mathbf{P}_{r r}\right) \boldsymbol{w}_{r} .
\end{aligned}
$$

Equivalently,

$$
\begin{aligned}
\boldsymbol{Q}_{\omega t} & =\mathbf{M}_{t r}^{d} \dot{\mathbf{P}}_{r r} \boldsymbol{w}_{r}+2 \mathbf{M}_{t f} \dot{\mathbf{P}}_{f f} \boldsymbol{w}_{f}, \\
\boldsymbol{Q}_{\omega r} & =\mathbf{P}_{r r}^{T} \mathbf{M}_{r r}^{d} \dot{\mathbf{P}}_{r r} \boldsymbol{w}_{r}+2 \mathbf{P}_{r r}^{T} \mathbf{M}_{r} f^{d} \dot{\mathbf{P}}_{f f} \boldsymbol{w}_{f}, \\
\boldsymbol{Q}_{\omega f} & =\mathbf{P}_{f f}^{T}\left(\mathbf{M}_{r} f^{d}\right)^{T} \dot{\mathbf{P}}_{r r} \boldsymbol{w}_{r}+2 \mathbf{P}_{f f}^{T} \mathbf{M}_{f f} \dot{\mathbf{P}}_{f f} \boldsymbol{w}_{f},
\end{aligned}
$$

or, in matrix notation,

$$
\left[\begin{array}{c}
\boldsymbol{Q}_{\omega t} \\
\boldsymbol{Q}_{\omega r} \\
\boldsymbol{Q}_{\omega f}
\end{array}\right]=\left[\begin{array}{ccc}
\mathbf{I} & \mathbf{O} & \mathbf{O} \\
\mathbf{O} & \mathbf{P}_{r r}^{T} & \mathbf{O} \\
\mathbf{O} & \mathbf{O} & \mathbf{P}_{f f}^{T}
\end{array}\right]\left[\begin{array}{ccc}
\mathbf{M}_{t t} & \mathbf{M}_{t r}^{d} & \mathbf{M}_{t f} \\
\cdot & \mathbf{M}_{r r}^{d} & \mathbf{M}_{r} f^{d} \\
\operatorname{sym} & \cdot & \mathbf{M}_{f f}
\end{array}\right]\left[\begin{array}{ccc}
\mathbf{O} & \mathbf{O} & \mathbf{O} \\
\mathbf{O} & \dot{\mathbf{P}}_{r r} & \mathbf{O} \\
\mathbf{O} & \mathbf{O} & 2 \dot{\mathbf{P}}_{f f}
\end{array}\right]\left[\begin{array}{c}
\boldsymbol{w}_{t} \\
\boldsymbol{w}_{r} \\
\boldsymbol{w}_{f}
\end{array}\right] .
$$

Subsequently, each term is being evaluated further. Doing so, the first term of $\boldsymbol{Q}_{\omega t}$ becomes

$$
\mathbf{M}_{t r}^{d} \dot{\mathbf{P}}_{r r} \boldsymbol{w}_{r}=m r^{i} \tilde{\boldsymbol{\omega}}^{2} \boldsymbol{d}_{i}=m \tilde{\boldsymbol{\omega}}^{2} \boldsymbol{r}=-m \tilde{\boldsymbol{\omega}} \tilde{\boldsymbol{r}} \boldsymbol{\omega},
$$

and the second one

$$
\left(\mathbf{M}_{t f} \boldsymbol{w}_{f}\right) \mathbf{P}_{r r} \boldsymbol{w}_{r}=-m b_{\mu}^{i} w_{f, \mu} \tilde{\boldsymbol{d}}_{i} \boldsymbol{\omega}=-m \widetilde{\mathbf{B} \boldsymbol{w}_{f}} \boldsymbol{\omega}=m \tilde{\boldsymbol{\omega}} \mathbf{B} \boldsymbol{w}_{f}
$$

Further, the first term of $\boldsymbol{Q}_{\omega r}$ obtains a common shape,

$$
\mathbf{P}_{r r}^{T} \mathbf{M}_{r r}^{d} \dot{\mathbf{P}}_{r r} \boldsymbol{w}_{r}=\boldsymbol{\omega} \times(\boldsymbol{\Theta} \boldsymbol{\omega})=\tilde{\boldsymbol{\omega}} \boldsymbol{\Theta}_{r r} \boldsymbol{\omega} .
$$

Proof Here, the calculations are given explicitly for the constant part of $\mathbf{M}_{r r}^{d}$, i.e. for $\mathbf{M}_{r r}$. For the additional terms in (1652), exactly the same arguments apply. Making use of (103) and (121), the left-hand side of (175) becomes

$$
\begin{aligned}
& \mathbf{P}_{r r}^{T} \mathbf{M}_{r r} \dot{\mathbf{P}}_{r r} \boldsymbol{w}_{r}=\left[\begin{array}{lll}
\tilde{\boldsymbol{d}}_{1} & \tilde{\boldsymbol{d}}_{2} & \tilde{\boldsymbol{d}}_{3}
\end{array}\right]\left[\begin{array}{ccc}
\vartheta^{11} \mathbf{I} & \cdot & \cdot \\
\cdot & \cdot & \cdot \\
\cdot & \cdot & \cdot
\end{array}\right]\left[\begin{array}{c}
\tilde{\boldsymbol{\omega}} \dot{\boldsymbol{d}}_{1} \\
\tilde{\boldsymbol{\omega}} \dot{\boldsymbol{d}}_{2} \\
\tilde{\boldsymbol{\omega}} \dot{\boldsymbol{d}}_{3}
\end{array}\right] \\
& =\vartheta^{i j} \tilde{\boldsymbol{d}}_{i} \tilde{\boldsymbol{\omega}} \dot{\boldsymbol{d}}_{j}=\vartheta^{i j} \boldsymbol{d}_{i} \times\left(\boldsymbol{\omega} \times \dot{\boldsymbol{d}}_{j}\right) \\
& =\vartheta^{i j}\left[\left(\boldsymbol{d}_{i} \cdot \dot{\boldsymbol{d}}_{j}\right) \boldsymbol{\omega}-\left(\boldsymbol{d}_{i} \cdot \boldsymbol{\omega}\right) \dot{\boldsymbol{d}}_{j}\right]=-\vartheta^{i j}\left(\boldsymbol{d}_{i} \cdot \boldsymbol{\omega}\right) \dot{\boldsymbol{d}}_{j} .
\end{aligned}
$$

The term $\vartheta^{i j} \boldsymbol{d}_{i} \cdot \dot{\boldsymbol{d}}_{j}$ vanishes since $\vartheta^{i j}$ is symmetric and $\boldsymbol{d}_{i} \cdot \dot{\boldsymbol{d}}_{j}$ is antisymmetric with respect to $i$ and $j$. On the other hand, Eq. (133) implies

$$
\boldsymbol{\Theta} \omega=\vartheta^{i i} \omega-\vartheta^{i j}\left(\boldsymbol{d}_{j} \cdot \boldsymbol{\omega}\right) \boldsymbol{d}_{i} .
$$

Inserting into the right-hand side of (175) yields

$$
\omega \times(\boldsymbol{\Theta} \omega)=\vartheta^{i i} \omega \times \omega-\vartheta^{i j}\left(\boldsymbol{d}_{j} \cdot \boldsymbol{\omega}\right) \boldsymbol{\omega} \times \boldsymbol{d}_{i}=-\vartheta^{i j}\left(\boldsymbol{d}_{j} \cdot \boldsymbol{\omega}\right) \dot{\boldsymbol{d}}_{i} .
$$


The equality of the right-hand sides of (176) and of (178), recall the symmetry of $\vartheta^{i j}$, completes the proof.

The second term of $\boldsymbol{Q}_{\omega r}$ becomes

$$
\begin{aligned}
\mathbf{P}_{r r}^{T}\left(\mathbf{M}_{r} f^{d} \boldsymbol{w}_{f}\right) \mathbf{P}_{r r} \boldsymbol{w}_{r} & =w_{f, \mu} \mathbf{P}_{r r}^{T} \mathbf{M}_{\mu}^{d} \mathbf{P}_{r r} \boldsymbol{w}_{r} \\
& =w_{f, \mu} \boldsymbol{\Theta}_{\mu}^{d} \boldsymbol{w}_{r}=\mathbf{L}_{r} f\left(\boldsymbol{\omega}, \boldsymbol{q}_{f}^{\prime}\right) \boldsymbol{w}_{f}
\end{aligned}
$$

with $\mathbf{M}_{\mu}^{d}\left(\boldsymbol{q}_{f}^{\prime}\right):=\mathbf{M}_{\mu}+q_{f, \nu}^{\prime} \mathbf{M}_{\mu \nu}$ and $\boldsymbol{\Theta}_{\mu}^{d}\left(\boldsymbol{q}_{f}^{\prime}\right):=\boldsymbol{\Theta}_{\mu}+q_{f, \nu}^{\prime} \boldsymbol{\Theta}_{\mu \nu}$. For notational convenience, a generalized angular momentum matrix $(3 \times M)$ has been introduced,

$$
\mathbf{L}_{r} f\left(\boldsymbol{\omega}, \boldsymbol{q}_{f}^{\prime}\right):=\left[\boldsymbol{\Theta}_{1}^{d} \boldsymbol{\omega} \cdots \boldsymbol{\Theta}_{M}^{d} \boldsymbol{\omega}\right] .
$$

Similarly, for the first term of $\boldsymbol{Q}_{\omega f}$,

$$
\begin{aligned}
\mathbf{P}_{f f}^{T}\left(\mathbf{M}_{r} f^{d}\right)^{T} \dot{\mathbf{P}}_{r r} \boldsymbol{w}_{r} & =\left[\begin{array}{c}
\boldsymbol{p}_{f f}^{T}\left(\mathbf{M}_{1}^{d}\right)^{T} \dot{\mathbf{P}}_{r r} \boldsymbol{w}_{r} \\
\vdots \\
\boldsymbol{p}_{f f}^{T}\left(\mathbf{M}_{M}^{d}\right)^{T} \dot{\mathbf{P}}_{r r} \boldsymbol{w}_{r}
\end{array}\right]=-\left[\begin{array}{c}
\boldsymbol{\omega}^{T}\left(\boldsymbol{\Theta}_{1}^{d}\right)^{T} \boldsymbol{\omega} \\
\vdots \\
\omega^{T}\left(\boldsymbol{\Theta}_{M}^{d}\right)^{T} \boldsymbol{\omega}
\end{array}\right] . \\
& =-\mathbf{L}_{r} f^{T} \boldsymbol{\omega}
\end{aligned}
$$

The latter equality follows from the definition of the generalized inertia tensors $\boldsymbol{\Theta}_{\mu}$ and $\boldsymbol{\Theta}_{\mu \nu}$, Eqs. (136) and (138), respectively, since

$$
\begin{aligned}
\boldsymbol{p}_{f f}^{T} \mathbf{M}_{\mu}^{T} \dot{\mathbf{P}}_{r r} \boldsymbol{w}_{r} & =\vartheta_{\mu}^{j i} \boldsymbol{d}_{i}^{T} \tilde{\boldsymbol{\omega}} \dot{\boldsymbol{d}}_{j}=\vartheta_{\mu}^{j i} \boldsymbol{d}_{i} \cdot\left[\boldsymbol{\omega} \times\left(\boldsymbol{\omega} \times \boldsymbol{d}_{j}\right)\right] \\
& =\vartheta_{\mu}^{j i}\left[\left(\boldsymbol{\omega} \cdot \boldsymbol{d}_{i}\right)\left(\boldsymbol{\omega} \cdot \boldsymbol{d}_{j}\right)-\boldsymbol{\omega}^{2}\left(\boldsymbol{d}_{i} \cdot \boldsymbol{d}_{j}\right)\right]=-\boldsymbol{\omega} \cdot\left(\boldsymbol{\Theta}_{\mu}^{T} \boldsymbol{\omega}\right),
\end{aligned}
$$

and analogously for $\mathbf{M}_{\mu \nu}$.

Finally, the second term of $\boldsymbol{Q}_{\omega f}$ becomes

$$
\begin{aligned}
\mathbf{P}_{f f}^{T} \mathbf{M}_{f f} \dot{\mathbf{P}}_{f f} & =\left[\begin{array}{cccc}
\boldsymbol{p}_{f f}^{T} \mathbf{M}_{11} \dot{\boldsymbol{p}}_{f f} & \ldots & \boldsymbol{p}_{f f}^{T} \mathbf{M}_{1 M} \dot{\boldsymbol{p}}_{f f} \\
\vdots & \ddots & \vdots \\
\boldsymbol{p}_{f f}^{T} \mathbf{M}_{M 1} \dot{\boldsymbol{p}}_{f f} & \ldots & \boldsymbol{p}_{f f}^{T} \mathbf{M}_{M M} \dot{\boldsymbol{p}}_{f f}
\end{array}\right] \\
& =\left[\begin{array}{cccc}
0 & -\boldsymbol{\omega}^{T} \boldsymbol{\vartheta}_{12} & \ldots & -\boldsymbol{\omega}^{T} \boldsymbol{\vartheta}_{1 M} \\
\boldsymbol{\omega}^{T} \boldsymbol{\vartheta}_{12} & 0 & \ldots & -\boldsymbol{\omega}^{T} \boldsymbol{\vartheta}_{2 M} \\
\vdots & \vdots & \ddots & \vdots \\
\boldsymbol{\omega}^{T} \boldsymbol{\vartheta}_{1 M} & \boldsymbol{\omega}^{T} \boldsymbol{\vartheta}_{2 M} & \ldots & 0
\end{array}\right]=: \mathbf{L}_{f f}(\boldsymbol{\omega})
\end{aligned}
$$

involving an additional $M \times M$ momentum matrix $\mathbf{L}_{f f}$. Use has been made of the definition of inertia vectors (147),

$$
\boldsymbol{p}_{f f} \mathbf{M}_{\mu \nu}^{T} \dot{\boldsymbol{p}}_{f f}=\vartheta_{\mu \nu}^{i j} \boldsymbol{d}_{i}^{T} \dot{\boldsymbol{d}}_{j}=\vartheta_{\mu \nu}^{i j} \boldsymbol{d}_{i} \cdot\left(\boldsymbol{\omega} \times \boldsymbol{d}_{j}\right)=-\boldsymbol{\omega} \cdot \boldsymbol{\vartheta}_{\mu \nu} .
$$

In particular, for one single flexible mode, i.e. $M=1$, one has $\mathbf{L}_{f f}=[0]$ and thus $\mathbf{P}_{f f}^{T} \mathbf{M}_{f f} \dot{\mathbf{P}}_{f f}=[0]$.

Concluding, the generalized gyroscopic forces read

$$
\left[\begin{array}{l}
\boldsymbol{Q}_{\omega t} \\
\boldsymbol{Q}_{\omega r} \\
\boldsymbol{Q}_{\omega f}
\end{array}\right]=\left[\begin{array}{cc}
-m \tilde{\boldsymbol{\omega}} \tilde{\boldsymbol{r}} & m \tilde{\boldsymbol{\omega}} \mathbf{B} \\
\tilde{\boldsymbol{\omega}} \boldsymbol{\Theta}_{r r} & \mathbf{L}_{r} f \\
-\mathbf{L}_{r} f^{T} & \mathbf{L}_{f f}
\end{array}\right]\left[\begin{array}{c}
\boldsymbol{\omega} \\
2 w_{f}
\end{array}\right]
$$

In particular, for one single flexible mode,

$$
\left[\begin{array}{c}
\boldsymbol{Q}_{\omega t} \\
\boldsymbol{Q}_{\omega r} \\
Q_{\omega f}
\end{array}\right]=\left[\begin{array}{cc}
-m \tilde{\boldsymbol{\omega}} \tilde{\boldsymbol{r}} & m \tilde{\boldsymbol{\omega}} \boldsymbol{b}_{1} \\
\tilde{\boldsymbol{\omega}} \boldsymbol{\Theta}_{r r} & \boldsymbol{\Theta}_{1}^{d} \boldsymbol{\omega} \\
-\left(\boldsymbol{\Theta}_{1}^{d} \boldsymbol{\omega}\right)^{T} & 0
\end{array}\right]\left[\begin{array}{c}
\boldsymbol{\omega} \\
2 w_{f}
\end{array}\right]
$$


As for the inertial forces, the coefficient matrices $\boldsymbol{r}, \mathbf{B}, \boldsymbol{\Theta}_{r r}, \boldsymbol{\Theta}_{\mu}, \boldsymbol{\Theta}_{\mu \nu}$, and $\boldsymbol{\vartheta}_{\mu \nu}$ evolve according to a rigid body rotation, i.e.

$$
\begin{array}{rlrlrl}
\boldsymbol{r} & =\mathbf{A} \overline{\boldsymbol{r}}, & \mathbf{B} & =\mathbf{A} \overline{\mathbf{B}}, & \boldsymbol{\vartheta}_{\mu \nu} & =\mathbf{A} \overline{\boldsymbol{\vartheta}}_{\mu \nu}, \\
\boldsymbol{\Theta}_{r r} & =\mathbf{A} \overline{\boldsymbol{\Theta}}_{r r} \mathbf{A}^{T}, \boldsymbol{\Theta}_{\mu}=\mathbf{A} \overline{\boldsymbol{\Theta}}_{\mu} \mathbf{A}^{T}, & \boldsymbol{\Theta}_{\mu \nu} & =\mathbf{A} \overline{\boldsymbol{\Theta}}_{\mu \nu} \mathbf{A}^{T},
\end{array}
$$

with constant quantities $\overline{\mathbf{B}}, \bar{\vartheta}_{\mu \nu}, \overline{\boldsymbol{\Theta}}_{\mu}, \overline{\boldsymbol{\Theta}}_{\mu \nu}$, and $\overline{\boldsymbol{r}}$ as well as $\overline{\boldsymbol{\Theta}}_{r r}$ only depending on the flexible coordinates $q_{\mu}^{\prime}$.

\subsection{Elastic forces}

After projection, the generalized elastic forces read

$$
\boldsymbol{Q}_{e r}=\mathbf{P}_{r r}^{T} \hat{\mathbf{K}}_{r} f \mathbf{P}_{f f} \boldsymbol{q}_{f}^{\prime}+\mathbf{P}_{r} f^{T} \hat{\mathbf{K}}_{f f} \mathbf{P}_{f f} \boldsymbol{q}_{f}^{\prime} \doteq \mathbf{P}_{r r}^{T} \mathbf{A}_{\mathrm{bd}} \overline{\mathbf{K}}_{r} f \mathbf{A}_{\mathrm{bd}}^{T} \mathbf{P}_{f f} \boldsymbol{q}_{f}^{\prime}
$$

and

$$
\boldsymbol{Q}_{e f}=\mathbf{P}_{f f}^{T} \hat{\mathbf{K}}_{f f} \mathbf{P}_{f f} \boldsymbol{q}_{f}^{\prime}=\mathbf{P}_{f f}^{T} \mathbf{A}_{\mathrm{bd}} \overline{\mathbf{K}}_{f f} \mathbf{A}_{\mathrm{bd}}^{T} \mathbf{P}_{f f} \boldsymbol{q}_{f}^{\prime}
$$

where in each case $\mathbf{A}_{\mathrm{bd}}$ denotes an appropriate block diagonal rotation matrix. Note that the term involving $\mathbf{P}_{r} f=\boldsymbol{q}_{f}^{\prime} \otimes \mathbf{P}_{r r}$ in (187) is quadratic in $\boldsymbol{q}_{f}^{\prime}$. It drops out since the present theory is developed under the assumption of small flexible deformations. One has

$$
\mathbf{P}_{r r}^{T} \mathbf{A}_{\mathrm{bd}}=\left[\begin{array}{lll}
\tilde{\boldsymbol{d}}_{1} & \tilde{\boldsymbol{d}}_{2} & \tilde{\boldsymbol{d}}_{3}
\end{array}\right]\left[\begin{array}{ccc}
\mathbf{A} & \mathbf{O} & \mathbf{0} \\
\mathbf{O} & \mathbf{A} & \mathbf{O} \\
\mathbf{O} & \mathbf{O} & \mathbf{A}
\end{array}\right]=\mathbf{A}\left[\begin{array}{lll}
\tilde{\boldsymbol{e}}_{1} & \tilde{\boldsymbol{e}}_{2} & \tilde{\boldsymbol{e}}_{3}
\end{array}\right] \stackrel{\dot{=}}{=} \mathbf{A} \tilde{\mathbf{I}}
$$

Further, for one single flexible mode,

$$
\mathbf{A}_{\mathrm{bd}}^{T} \boldsymbol{p}_{f f}=\left[\begin{array}{ccc}
\mathbf{A}^{T} & \mathbf{O} & \mathbf{O} \\
\mathbf{O} & \mathbf{A}^{T} & \mathbf{O} \\
\mathbf{O} & \mathbf{O} & \mathbf{A}^{T}
\end{array}\right]\left[\begin{array}{l}
\boldsymbol{d}_{1} \\
\boldsymbol{d}_{2} \\
\boldsymbol{d}_{3}
\end{array}\right]=\left[\begin{array}{l}
\boldsymbol{e}_{1} \\
\boldsymbol{e}_{2} \\
\boldsymbol{e}_{3}
\end{array}\right]=: \boldsymbol{i} \in \mathbb{R}^{9 \times 1}
$$

In the general case,

$$
\mathbf{A}_{\mathrm{bd}}^{T} \mathbf{P}_{f f}=\left[\begin{array}{cccc}
\boldsymbol{i} & \mathbf{0} & \ldots & \mathbf{0} \\
\mathbf{0} & \boldsymbol{i} & \ldots & \mathbf{0} \\
\vdots & \vdots & \ddots & \vdots \\
\mathbf{0} & \mathbf{0} & \ldots & \boldsymbol{i}
\end{array}\right] \stackrel{\doteq}{=} \mathbf{I}_{\mathrm{bd}}^{T} \in \mathbb{R}^{9 M \times M}
$$

and thus

$$
\boldsymbol{Q}_{e f}=\mathbf{I}_{\mathrm{bd}} \overline{\mathbf{K}}_{f f} \mathbf{I}_{\mathrm{bd}}^{T} \boldsymbol{q}_{f}^{\prime}=\mathbf{K}_{f f} \boldsymbol{q}_{f}^{\prime}
$$

In fact, the constant, reduced $(M \times M)$ stiffness matrix

$$
\mathbf{K}_{f f}=\mathbf{I}_{\mathrm{bd}} \overline{\mathbf{K}}_{f f} \mathbf{I}_{\mathrm{bd}}^{T},
$$

turns out to coincide with the FFRF stiffness matrix. The elastic forces related to the rotational degrees of freedom have to vanish,

$$
\boldsymbol{Q}_{e r}=\mathbf{A} \tilde{\mathbf{I}} \overline{\mathbf{K}}_{r} f \mathbf{I}_{\mathrm{bd}}^{T} \boldsymbol{q}_{f}^{\prime}=\mathbf{0},
$$

since the latter now exactly represent rigid body motions. A general algebraic proof of (194) is not trivial. In the Appendix, it is shown that

$$
\tilde{\mathbf{I}} \overline{\mathbf{K}}_{r} f=\tilde{\mathbf{I}} \boldsymbol{\Phi}_{r}^{T} \mathbf{K} \boldsymbol{\Phi}_{f}=\mathbf{O}
$$

using the example of a truss structure. Since any volume element can be approximated by a framework consisting of truss elements, it is concluded that (195) is at least a good approximation, in general. This assumption is confirmed by numerical experiments which have shown that the largest elements of $\tilde{\mathbf{I}} \overline{\mathbf{K}}_{r} f$ are by a factor of at least $10^{-9}$ smaller than the ones of $\mathbf{K}_{f f}$. 
7.11 Nonlinear forces

Subsequently, it will be shown that the nonlinear forces $\hat{f}_{\text {nl }}$, which are characteristic for the GCMS formulation, disappear in the course of the null-space projection. Inserting (82 $)$ and (95) into (61),

$$
\hat{\boldsymbol{f}}_{\mathrm{nl}}^{T}=\boldsymbol{q}_{f}^{\prime}{ }^{T} \mathbf{P}_{f f}^{T} \mathbf{A}_{\mathrm{bd}} \hat{\mathbf{K}}_{f f} \frac{\partial \mathbf{A}_{\mathrm{bd}}^{T}}{\partial \boldsymbol{q}_{r}} \mathbf{P}_{f f} \boldsymbol{q}_{f}^{\prime}=\mathbf{0}^{(1 \times 9)}
$$

since

$$
\frac{\partial \mathbf{A}_{\mathrm{bd}}^{T}}{\partial \boldsymbol{q}_{r}} \mathbf{P}_{f f} \boldsymbol{q}_{f}^{\prime}=\frac{\partial \mathbf{A}_{\mathrm{bd}}^{T} \mathbf{P}_{f f}}{\partial \boldsymbol{q}_{r}} \boldsymbol{q}_{f}^{\prime}-\mathbf{A}_{\mathrm{bd}}^{T} \frac{\partial \mathbf{P}_{f f}}{\partial \boldsymbol{q}_{r}} \boldsymbol{q}_{f}^{\prime}=\mathbf{O}^{(9 M \times 9)} .
$$

The first term vanishes, since $\mathbf{A}_{\mathrm{bd}}^{T} \mathbf{P}_{f f}=\mathbf{I}_{\mathrm{bd}}^{T}$ is constant, and the second one since the GCMS coordinates $\boldsymbol{q}_{r}$ and $\boldsymbol{q}_{f}$ are independent of each other,

$$
\frac{\partial \mathbf{P}_{f f}}{\partial \boldsymbol{q}_{r}} \boldsymbol{q}_{f}^{\prime}=\frac{\partial \mathbf{P}_{f f} \boldsymbol{q}_{f}^{\prime}}{\partial \boldsymbol{q}_{r}}=\frac{\partial \boldsymbol{q}_{f}}{\partial \boldsymbol{q}_{r}}=\mathbf{O}^{(9 M \times 9)}
$$

\subsection{External forces}

The projected external forces become

$$
\boldsymbol{Q}_{\mathrm{ext}}^{\prime}=\left[\begin{array}{c}
\boldsymbol{\Phi}_{t}^{T} \\
\left(\boldsymbol{\Phi}_{r} \mathbf{P}_{r r}\right)^{T}+\left(\boldsymbol{\Phi}_{f} \mathbf{P}_{f r}\right)^{T} \\
\left(\boldsymbol{\Phi}_{f} \mathbf{P}_{f f}\right)^{T}
\end{array}\right] \boldsymbol{f}_{\mathrm{ext}} \doteq\left[\begin{array}{c}
\boldsymbol{F}_{t} \\
\mathbf{A} \bar{M} \\
\boldsymbol{F}_{f}
\end{array}\right]
$$

Following the same argument as in the previous subsection, the term involving $\mathbf{P}_{f r}$ drops out in the present stage of approximation, since it involves small quantities of second order (deformational amplitudes and forces). Explicitly, the resultant force vector

$$
\boldsymbol{F}_{t}=\sum_{n=1}^{N} \boldsymbol{f}_{n}
$$

the resultant moment vector

$$
\boldsymbol{M}=\sum_{n=1}^{N} x_{n}^{i} \boldsymbol{d}_{i} \times \boldsymbol{f}_{n}=\sum_{n=1}^{N}\left(\mathbf{A} \overline{\boldsymbol{x}}_{n}\right) \times \boldsymbol{f}_{n}=\mathbf{A} \sum_{n=1}^{N} \overline{\boldsymbol{x}}_{n} \times \overline{\boldsymbol{f}}_{n}=\mathbf{A} \overline{\boldsymbol{M}},
$$

and the vector of the reduced flexible forces $\mathbf{F}_{f}=\left[\begin{array}{lll}F_{f, 1} & \ldots & F_{f, M}\end{array}\right]^{T}$, where

$$
\begin{aligned}
F_{f, \mu} & =\sum_{n=1}^{N} \phi_{f, \mu(3(n-1)+i)} \boldsymbol{d}_{i} \cdot \boldsymbol{f}_{n} \\
& =\sum_{n=1}^{N}\left(\mathbf{A} \overline{\boldsymbol{\phi}}_{f, \mu}\left(\overline{\boldsymbol{x}}_{n}\right)\right) \cdot \boldsymbol{f}_{n}=\sum_{n=1}^{N} \overline{\boldsymbol{\phi}}_{f, \mu}\left(\overline{\boldsymbol{x}}_{n}\right) \cdot \overline{\boldsymbol{f}}_{n} .
\end{aligned}
$$

Therein, $\boldsymbol{f}_{n}$ is the force vector applied at node $n, \overline{\boldsymbol{f}}_{n}$ its co-rotational counterpart, and $\overline{\boldsymbol{\phi}}_{f, \mu}\left(\overline{\boldsymbol{x}}_{n}\right)=$ $\phi_{f, \mu(3(n-1)+i)} \boldsymbol{e}_{i}$ the co-rotational deflection of the $\mu$ th flexible mode at the position of the $n$th node. 


\section{Projected equations of motion}

Inserting the generalized accelerations (101) into the equations of motion (87), multiplying with the total projection matrix (73) from the left and considering the results of the previous section, one obtains

$$
\begin{aligned}
& {\left[\begin{array}{ccc}
m \mathbf{I} & m \tilde{\boldsymbol{r}} & m \mathbf{B} \\
\cdot & \boldsymbol{\Theta}_{r r} & \boldsymbol{\Theta}_{r f} \\
\operatorname{sym} & \cdot & \boldsymbol{\Theta}_{f f}
\end{array}\right]\left[\begin{array}{c}
\ddot{\boldsymbol{q}}_{t} \\
\dot{\boldsymbol{\omega}} \\
\ddot{\boldsymbol{q}}_{f}^{\prime}
\end{array}\right]+\left[\begin{array}{cc}
-m \tilde{\boldsymbol{\omega}} \tilde{\boldsymbol{r}} & m \tilde{\boldsymbol{\omega}} \mathbf{B} \\
\tilde{\boldsymbol{\omega}} \boldsymbol{\Theta}_{r r} & \mathbf{L}_{r} f \\
-\mathbf{L}_{r} f^{T} & \mathbf{L}_{f f}
\end{array}\right]\left[\begin{array}{c}
\boldsymbol{\omega} \\
2 \dot{\boldsymbol{q}}_{f}^{\prime}
\end{array}\right]+\left[\begin{array}{c}
\mathbf{O} \\
\mathbf{O} \\
\mathbf{K}_{f f}
\end{array}\right] \boldsymbol{q}_{f}^{\prime}} \\
& +\mathbf{C}^{\prime T} \boldsymbol{\lambda}=\left[\begin{array}{c}
\boldsymbol{F}_{t} \\
\boldsymbol{M} \\
\boldsymbol{F}_{f}
\end{array}\right] .
\end{aligned}
$$

Summarizing, the non-constant inertia coefficients in (203) can be computed via $\mathbf{B}\left(\boldsymbol{q}_{r}\right)=\mathbf{A} \overline{\mathbf{B}}$ and

$$
\begin{aligned}
m \boldsymbol{r}\left(\boldsymbol{q}_{r}, \boldsymbol{q}_{f}^{\prime}\right) & =m \mathbf{A} \overline{\boldsymbol{r}}, & \overline{\boldsymbol{r}}\left(\boldsymbol{q}_{f}^{\prime}\right) & =\overline{\boldsymbol{a}}-q_{f, \mu}^{\prime} \overline{\boldsymbol{b}}_{\mu}, \\
\boldsymbol{\Theta}_{r r}\left(\boldsymbol{q}_{r}, \boldsymbol{q}_{f}^{\prime}\right) & =\mathbf{A} \overline{\boldsymbol{\Theta}}_{r r} \mathbf{A}^{T}, & \overline{\boldsymbol{\Theta}}_{r r}\left(\boldsymbol{q}_{f}^{\prime}\right) & =\overline{\boldsymbol{\Theta}}^{d}+q_{f, \mu}^{\prime} \overline{\boldsymbol{\Theta}}_{\mu}^{d}, \\
\boldsymbol{\Theta}_{r} f\left(\boldsymbol{q}_{r}, \boldsymbol{q}_{f}^{\prime}\right) & =\mathbf{A} \overline{\boldsymbol{\Theta}}_{r} f \mathbf{A}^{T}, & \overline{\boldsymbol{\Theta}}_{r} f\left(\boldsymbol{q}_{f}^{\prime}\right) & =\overline{\boldsymbol{\Xi}}+q_{f, \mu}^{\prime} \overline{\boldsymbol{\Xi}}_{\mu},
\end{aligned}
$$

with

$$
\overline{\mathbf{\Theta}}^{d}=\overline{\mathbf{\Theta}}+q_{f, \mu}^{\prime} \overline{\mathbf{\Theta}}_{\mu}, \quad \overline{\mathbf{\Theta}}_{\mu}^{d}=\overline{\mathbf{\Theta}}_{\mu}+q_{f, v}^{\prime} \overline{\mathbf{\Theta}}_{\mu \nu}
$$

from the constant inertia components

$$
m \overline{\boldsymbol{a}}^{T}=\boldsymbol{\phi}_{t}^{T} \mathbf{M} \boldsymbol{\phi}_{t}, \quad m \overline{\boldsymbol{b}}_{\mu}^{T}=\boldsymbol{\phi}_{t}^{T} \mathbf{M} \boldsymbol{\phi}_{f, \mu}
$$

as well as

$$
\begin{aligned}
& \overline{\boldsymbol{\Theta}}=(\operatorname{trace} \overline{\mathbf{T}}) \mathbf{I}-\overline{\mathbf{T}}, \quad \overline{\mathbf{T}}=\boldsymbol{\phi}_{r}^{T} \mathbf{M} \boldsymbol{\phi}_{r}, \\
& \overline{\boldsymbol{\Theta}}_{\mu}=\left(\operatorname{trace} \overline{\mathbf{T}}_{\mu}\right) \mathbf{I}-\overline{\mathbf{T}}_{\mu}, \quad \overline{\mathbf{T}}_{\mu}=\boldsymbol{\phi}_{r}^{T} \mathbf{M} \boldsymbol{\phi}_{f, \mu}, \\
& \overline{\boldsymbol{\Theta}}_{\mu \nu}=\left(\operatorname{trace} \overline{\mathbf{T}}_{\mu \nu}\right) \mathbf{I}-\overline{\mathbf{T}}_{\mu \nu}, \overline{\mathbf{T}}_{\mu \nu}=\boldsymbol{\phi}_{f, \mu}^{T} \mathbf{M} \boldsymbol{\phi}_{f, \nu}
\end{aligned}
$$

and $^{4}$

$$
\begin{aligned}
\overline{\mathbf{B}} & =\left[\begin{array}{llll}
\overline{\boldsymbol{b}}_{1} & \cdots & \overline{\boldsymbol{b}}_{M}
\end{array}\right], & \overline{\boldsymbol{\Theta}}_{f f} & =\left[\operatorname{trace} \overline{\mathbf{T}}_{\mu \nu}\right]=\boldsymbol{\Theta}_{f f}, \\
\overline{\boldsymbol{\Xi}} & =\left[\begin{array}{llll}
\overline{\boldsymbol{\vartheta}}_{1} & \cdots & \overline{\boldsymbol{\vartheta}}_{M}
\end{array}\right], & \overline{\boldsymbol{\vartheta}}_{\mu} & =\operatorname{ax}\left(\overline{\boldsymbol{\Theta}}_{\mu}-\overline{\boldsymbol{\Theta}}_{\mu}^{T}\right), \\
\overline{\boldsymbol{\Xi}}_{\mu} & =\left[\begin{array}{llll}
\overline{\boldsymbol{\vartheta}}_{\mu 1} & \cdots & \overline{\boldsymbol{\vartheta}}_{\mu M}
\end{array}\right], & \overline{\boldsymbol{\vartheta}}_{\mu \nu} & =\operatorname{ax}\left(\overline{\boldsymbol{\Theta}}_{\mu \nu}-\overline{\boldsymbol{\Theta}}_{\mu \nu}^{T}\right),
\end{aligned}
$$

which are all determined uniquely by the constant FE mass matrix $\mathbf{M}$, the nodal coordinates of the initial configuration (entering $\boldsymbol{\phi}_{r}$ ), and the flexible mode shapes (entering $\boldsymbol{\phi}_{f, \mu}$ ). Finally, the generalized angular momentum components entering the gyroscopic terms are given by

$$
\begin{aligned}
\mathbf{L}_{r} f\left(\boldsymbol{\omega}, \boldsymbol{q}_{r}, \boldsymbol{q}_{f}^{\prime}\right) & =\mathbf{A}\left[\overline{\boldsymbol{\Theta}}_{1}^{d} \mathbf{A}^{T} \boldsymbol{\omega} \cdots \overline{\boldsymbol{\Theta}}_{M}^{d} \mathbf{A}^{T} \boldsymbol{\omega}\right]=\mathbf{A} \overline{\mathbf{L}}_{r} f\left(\overline{\boldsymbol{\omega}}, \boldsymbol{q}_{f}^{\prime}\right), \\
\mathbf{L}_{f f}\left(\boldsymbol{\omega}, \boldsymbol{q}_{r}\right) & =\mathbf{A}\left[\boldsymbol{\omega}^{T} \overline{\boldsymbol{\vartheta}}_{\mu \nu}\right]=\left[\overline{\boldsymbol{\omega}}^{T} \overline{\boldsymbol{\vartheta}}_{\mu \nu}\right]=\overline{\mathbf{L}}_{f f}(\overline{\boldsymbol{\omega}}) .
\end{aligned}
$$

Note that in (203), the vector components $\boldsymbol{q}_{t}$ and $\boldsymbol{\omega}$ refer to the inertial frame $\left(\boldsymbol{e}_{1}, \boldsymbol{e}_{2}, \boldsymbol{e}_{3}\right)$, whereas the flexible coordinates $\boldsymbol{q}_{f}^{\prime}$ are non-vectorial quantities and thus independent of a coordinate system. Since the rotation matrix $\mathbf{A}$ is required for the update of the inertia components, a convenient parameterization of rotations has to be introduced. Using the common concept of Euler parameters [19], $\boldsymbol{q}_{r}^{\prime}=\left[\begin{array}{ll}\theta_{0} & \boldsymbol{\theta}\end{array}\right] \in \mathbb{R}^{4}$, the expressions

$$
\omega=\mathbf{G} \dot{\boldsymbol{q}}_{r}^{\prime}, \quad \dot{\omega}=\mathbf{G} \ddot{\boldsymbol{q}}_{r}^{\prime}
$$

with the $3 \times 4$ matrix

$$
\mathbf{G}=2\left[\begin{array}{ll}
-\boldsymbol{\theta} & \theta_{0} \mathbf{I}+\tilde{\boldsymbol{\theta}}
\end{array}\right]
$$

\footnotetext{
${ }^{4}$ The abbreviated notation $\left[(\cdot)_{\mu \nu}\right]$ stands for the corresponding $M \times M$ matrix.
} 
have to be substituted in (203). Considering

$$
\overline{\mathbf{G}}=2\left[\begin{array}{ll}
-\boldsymbol{\theta} & \theta_{0} \mathbf{I}-\tilde{\boldsymbol{\theta}}
\end{array}\right]=\mathbf{A}^{T} \mathbf{G}
$$

and

$$
\mathbf{A}=\frac{1}{4} \mathbf{G} \overline{\mathbf{G}}^{T}
$$

one obtains

$$
\begin{aligned}
& {\left[\begin{array}{ccc}
m \mathbf{I} & m \mathbf{A} \tilde{\overline{\boldsymbol{r}}} \overline{\mathbf{G}} & m \mathbf{A} \overline{\mathbf{B}} \\
\cdot & \overline{\mathbf{G}}^{T} \overline{\mathbf{\Theta}}_{r r} \overline{\mathbf{G}} & \overline{\mathbf{G}}^{T} \overline{\mathbf{\Theta}}_{r f} \\
\operatorname{sym} & \cdot & \overline{\boldsymbol{\Theta}}_{f f}
\end{array}\right]\left[\begin{array}{c}
\ddot{\boldsymbol{q}}_{t} \\
\ddot{\boldsymbol{q}}_{r}^{\prime} \\
\ddot{\boldsymbol{q}}_{f}^{\prime}
\end{array}\right]+\left[\begin{array}{c}
\mathbf{O} \\
\mathbf{O} \\
\mathbf{K}_{f f}
\end{array}\right] \boldsymbol{q}_{f}^{\prime}+\mathbf{C}^{\prime T} \boldsymbol{\lambda}} \\
& =\left[\begin{array}{c}
\boldsymbol{F}_{t} \\
\overline{\mathbf{G}}^{T} \overline{\boldsymbol{M}} \\
\boldsymbol{F}_{f}
\end{array}\right]+\left[\begin{array}{c}
\boldsymbol{Q}_{v t} \\
\boldsymbol{Q}_{v r} \\
\boldsymbol{Q}_{v f}
\end{array}\right] .
\end{aligned}
$$

Apparently, the structure of Eq. (214) coincides with the one of the equations of motion of FFRF [19]. On closer inspection, a one-to-one correspondence can be established between the sub-matrices of the mass matrix in (214) and the inertia integrals characteristic for the standard FFRF. In the course of the null-space projection, the case-dependent constraint Jacobian, introduced in (88), gives rise to

$$
\mathbf{C}^{\prime}=\left[\begin{array}{ccc}
\mathbf{O} & \frac{\partial C_{r}}{\partial \boldsymbol{q}_{r}^{\prime}} & \mathbf{O} \\
\mathbf{C}_{b t} & \mathbf{C}_{b r} \mathbf{P}_{r r} \mathbf{G} & \mathbf{C}_{b f} \mathbf{P}_{f f}
\end{array}\right]
$$

which enters (214). The second block row of (215) refers to the single or multibody constraints, Eq. (62). According to the special choice of rotation parameters, the first block row constitutes the normalization condition for the Euler parameters

$$
C_{r}\left(\boldsymbol{q}_{r}^{\prime}\right) \equiv \frac{1}{2}\left(\theta_{0}^{2}+\boldsymbol{\theta}^{2}-1\right)=0 .
$$

The quadratic velocity vector on the right-hand side of (214) follows from the gyroscopic terms in (203) via the substitution of the angular velocity vector,

$$
\begin{aligned}
& \boldsymbol{Q}_{v t}=m \mathbf{A}\left(\widetilde{\overline{\mathbf{G}} \dot{\boldsymbol{q}}_{r}^{\prime}} \tilde{\overline{\boldsymbol{r}}} \overline{\mathbf{G}} \dot{\boldsymbol{q}}_{r}^{\prime}-2 \widetilde{\overline{\mathbf{G}} \dot{\boldsymbol{q}}_{r}^{\prime}} \overline{\mathbf{B}} \dot{\boldsymbol{q}}_{f}^{\prime}\right), \\
& \boldsymbol{Q}_{v r}=-\overline{\mathbf{G}}^{T}\left(\widetilde{\overline{\mathbf{G}} \dot{\boldsymbol{q}}_{r}^{\prime}} \overline{\boldsymbol{\Theta}}_{r r} \overline{\mathbf{G}} \dot{\boldsymbol{q}}_{r}^{\prime}+2 \overline{\mathbf{L}}_{r} f \dot{\boldsymbol{q}}_{f}^{\prime}\right), \\
& \boldsymbol{Q}_{v f}=\overline{\mathbf{L}}_{r} f^{T} \overline{\mathbf{G}} \dot{\boldsymbol{q}}_{r}^{\prime}-2 \overline{\mathbf{L}}_{f f} \dot{\boldsymbol{q}}_{f}^{\prime} .
\end{aligned}
$$

Despite its rather complicated shape, the numerical evaluation of (217) is straight forward and computationally inexpensive in the sense that it involves simple matrix multiplications only. This is in contrast with standard implementations of the FFRF, which require repeated evaluations of volume integrals. Note that all inertia coefficients in (214) and (217) depend—if at all—only on the small flexible amplitudes $q_{\mu}^{\prime}$ and are thus nearly constant in time!

\section{Discussion and conclusion}

The paper shows the interrelation of the absolute coordinates formulation (ACF) and the floating frame of reference formulation (FFRF). In particular, methods of modal reduction are addressed for both approaches. In a subsequent step, it is shown in detail how the modally reduced FFRF evolves from the modally reduced ACF (i.e. the GCMS) via a null-space projection approach. It turns out that the expressions for the inertia terms obtained in the course of the presented derivation have a favourable shape: All inertia terms can be computed from constant inertia coefficients, Eqs. $(204,205)$, which derive from the FE mass matrix via simple vector-matrix operations, Eqs. $(206,207)$. In view of a computer implementation, they can be provided in a preprocessing step. The same holds for the components of the quadratic velocity vector. Conventional implementations of the FFRF require the evaluation of so-called inertia integrals, which has to be performed on the algorithmic level of the finite element routine and thus strictly depend on the underlying FE code. In 
contrast, the presented formulation allows to run a FFRF simulation stand-alone. All required data (nodal coordinates, flexible mode shapes, global mass and stiffness matrix) are usually available via the common user interface of commercial FE software.

It is an interesting by-product of the presented derivations that the relation of the mass matrices of the GCMS and the FFRF can be given a very plausible shape, see Eq. (166). The same holds for the gyroscopic forces, Eq. (172).

\section{Appendix: Evaluation of the stiffness matrix}

Due to its simple shape, the stiffness matrix of a truss structure is well suited to demonstrate the role of the stiffness matrix in the course of the null-space projection. The essential circumstances can be illustrated with the help of a simple FE model consisting of three nodes with position vectors $\boldsymbol{r}_{1}, \boldsymbol{r}_{2}$, and $\boldsymbol{r}_{3}$ and two two-node truss elements connected at node two. The length of the trusses shall be $l_{1}$ and $l_{2}$, respectively, and their longitudinal stiffness $k_{1,2}=E_{1,2} A_{1,2} / l_{1,2}$. Then, the stiffness matrix of the structure reads [17]

$$
\mathbf{K}=\left[\begin{array}{ccc}
{[1.4] k_{1} \boldsymbol{t}_{1} \boldsymbol{t}_{1}^{T}} & -k_{1} \boldsymbol{t}_{1} \boldsymbol{t}_{1}^{T} & \mathbf{O} \\
-k_{1} \boldsymbol{t}_{1} \boldsymbol{t}_{1}^{T} & k_{1} \boldsymbol{t}_{1} \boldsymbol{t}_{1}^{T}+k_{2} \boldsymbol{t}_{2} \boldsymbol{t}_{2}^{T} & -k_{2} \boldsymbol{t}_{2} \boldsymbol{t}_{2}^{T} \\
\mathbf{O} & -k_{2} \boldsymbol{t}_{2} \boldsymbol{t}_{2}^{T} & k_{2} \boldsymbol{t}_{2} \boldsymbol{t}_{2}^{T^{T}}
\end{array}\right]
$$

with $\boldsymbol{t}_{1}=\left(\boldsymbol{r}_{1}-\boldsymbol{r}_{2}\right) / l_{1}$ and $\boldsymbol{t}_{2}=\left(\boldsymbol{r}_{2}-\boldsymbol{r}_{3}\right) / l_{2}$. Obviously, from (34), $\boldsymbol{\Phi}_{t}^{T} \mathbf{K}=\mathbf{O}$ and thus

$$
\mathbf{K}_{t *}=\boldsymbol{\Phi}_{t}^{T} \mathbf{K} \boldsymbol{\Phi}_{*}=\mathbf{O}
$$

for $*=t, r, f$. Further, (35) and (189) yield

$$
\tilde{\mathbf{I}} \boldsymbol{\Phi}_{r}^{T}=\left[\begin{array}{lll}
\tilde{\boldsymbol{r}}_{1} & \tilde{\boldsymbol{r}}_{2} & \tilde{\boldsymbol{r}}_{3}
\end{array}\right] .
$$

Consequently,

$$
\begin{aligned}
& \tilde{\mathbf{I}} \boldsymbol{\Phi}_{r}^{T} \mathbf{K}=\left[k_{1}\left(\tilde{\boldsymbol{r}}_{1}-\tilde{\boldsymbol{r}}_{2}\right) \boldsymbol{t}_{1} \boldsymbol{t}_{1}^{T} \quad k_{1}\left(-\tilde{\boldsymbol{r}}_{1}+\tilde{\boldsymbol{r}}_{2}\right) \boldsymbol{t}_{1} \boldsymbol{t}_{1}^{T}+k_{2}\left(\tilde{\boldsymbol{r}}_{2}-\tilde{\boldsymbol{r}}_{3}\right) \boldsymbol{t}_{2} \boldsymbol{t}_{2}^{T}\right. \\
& \left.k_{2}\left(-\tilde{\boldsymbol{r}}_{2}+\tilde{\boldsymbol{r}}_{3}\right) \boldsymbol{t}_{2} \boldsymbol{t}_{2}^{T}\right]
\end{aligned}
$$

and, since $\left(\tilde{\boldsymbol{r}}_{1}-\tilde{\boldsymbol{r}}_{2}\right) \boldsymbol{t}_{1}=l_{1} \boldsymbol{t}_{1} \times \boldsymbol{t}_{1}=\mathbf{0}$ and $\left(\tilde{\boldsymbol{r}}_{2}-\tilde{\boldsymbol{r}}_{3}\right) \boldsymbol{t}_{2}=l_{2} \boldsymbol{t}_{2} \times \boldsymbol{t}_{2}=\mathbf{0}$,

$$
\tilde{\mathbf{I}} \mathbf{K}_{r f}=\tilde{\mathbf{I}} \boldsymbol{\Phi}_{r}^{T} \mathbf{K} \boldsymbol{\Phi}_{f}=\mathbf{O} \text {. }
$$

Acknowledgements Open access funding provided by University of Innsbruck and Medical University of Innsbruck.

Open Access This article is distributed under the terms of the Creative Commons Attribution 4.0 International License (http:// creativecommons.org/licenses/by/4.0/), which permits unrestricted use, distribution, and reproduction in any medium, provided you give appropriate credit to the original author(s) and the source, provide a link to the Creative Commons license, and indicate if changes were made.

\section{References}

1. Belytschko, T., Hsieh, B.J.: Nonlinear transient finite element analysis with convected coordinates. Int. J. Numer. Methods Eng. 7, 255-271 (1973)

2. Betsch, P.: The discrete null space method for the energy consistent integration of constrained mechanical systems. Part I: holonomic constraints. Comput. Methods Appl. Mech. Eng. 194(50-52), 5159-5190 (2005)

3. Betsch, P., Leyendecker, S.: The discrete null space method for the energy consistent integration of constrained mechanical systems. Part II: multibody dynamics. Int. J. Numer. Methods Eng. 67(4), 499-552 (2006)

4. Brüls, O., Duysinx, P., Golinval, J.C.: The global modal parameterization for non-linear model-order reduction in flexible multibody dynamics. Int. J. Numer. Methods Eng. 69, 948-977 (2007)

5. César de Sá, J.M.A., Natal Jorge, R.M., Fontes Valente, R.A., Areias, P.M.A.: Development of shear locking-free shell elements using an enhanced assumed strain formulation. Int. J. Numer. Methods Eng. 53, 1721-1750 (2002)

6. Frischkorn, J., Reese, S.: A solid-beam finite element and non-linear constitutive modelling. Comput. Methods Appl. Mech. Eng. 265, 195-212 (2013) 
7. García de Jalón, J., Bayo, E.: Kinematic and Dynamic Simulation of Multibody Systems: The Real-Time Challenge. Springer, New York (1994)

8. Gerstmayr, J.: Strain tensors in the absolute nodal coordinate and the floating frame of reference formulation. Nonlinear Dyn. 34, 133-145 (2003)

9. Gerstmayr, J., Ambrósio, J.A.C.: Component mode synthesis with constant mass and stiffness matrices applied to flexible multibody systems. Int. J. Numer. Methods Eng. 73(11), 1518-1546 (2008)

10. Gerstmayr, J., Schöberl, J.: A 3D finite element method for flexible multibody systems. Multibody Syst. Dyn. 15(4), 305-320 (2006)

11. Humer, A., Gerstmayr, J.: Energy-momentum conserving time integration of modally reduced flexible multibody systems. In: Proceedings of the ASME 2013 IDETC/CIE Conference. Portland, USA (2013)

12. Lehner, M.: Modellreduktion in elastischen Mehrkörpersystemen. Ph.D. thesis, Universität Stuttgart (2007)

13. Liang, C.G., Lance, G.M.: A differentiable null space method for constrained dynamic analysis. J. Mech. Transm. Autom. Des. 109, 405-411 (1987)

14. Naets, F., Tamarozzi, T., Heirman, G.H.K., Desmet, W.: Real-time flexible multibody simulation with global modal parameterization. Multibody Syst. Dyn. 27, 267-284 (2012)

15. Orzechowski, G., Matikainen, M.K., Mikkola, A.M.: Inertia forces and shape integrals in the floating frame of reference formulation. Nonlinear Dyn. 88, 19531968 (2017)

16. Pechstein, A., Reischl, D., Gerstmayr, J.: A generalized component mode synthesis approach for flexible multibody systems with a constant mass matrix. J. Comput. Nonlinear Dyn. 8(1), 11,019 (2013)

17. Rao, S.S.: The Finite Element Method in Engineering, 6th edn. Elsevier, Amsterdam (2018)

18. Schwertassek, R., Wallrapp, O.: Dynamik Flexibler Mehrkrpersysteme. Springer, Wiesbaden (1999)

19. Shabana, A.A.: Dynamics of Multibody Systems, 3rd edn. Cambridge University Press, New York (2013)

20. Shabana, A.A., Schwertassek, R.: Equivalance of the floating frame of reference approach and finite element formulations. Int. J. Non Linear Mech. 33, 417-432 (1998)

21. Sherif, K., Nachbagauer, K.: A detailed derivation of the velocity-dependent inertia forces in the floating frame of reference formulation. J. Comput. Nonlinear Dyn. 9(044), 501 (2014)

22. Simmonds, J.G.: A Brief on Tensor Analysis, 2nd edn. Springer, New York (1994)

23. Vetyukov, Y.: Finite element modeling of Kirchhoff-Love shells as smooth material surfaces. ZAMM Zeitschrift für angewandte Mathematik und Mechanik 94, 150-163 (2014)

24. Wempner, G.A.: Finite elements, finite rotations and small strains. Int. J. Solids Struct. 5, 117-153 (1969)

25. Yakoub, Y., Shabana, A.A.: Three dimensional absolute nodal coordinate formulation for beam elements. ASME J. Mech. Des. 123, 606-621 (2001)

Publisher's Note Springer Nature remains neutral with regard to jurisdictional claims in published maps and institutional affiliations. 